Document downloaded from:

http://hdl.handle.net/10251/165757

This paper must be cited as:

Agud Albesa, L.; Calabuig, JM.; Sánchez Pérez, EA. (2020). Weighted p-regular kernels for reproducing kernel Hilbert spaces and Mercer Theorem. Analysis and Applications. 18(3):359-383. https://doi.org/10.1142/S0219530519500179

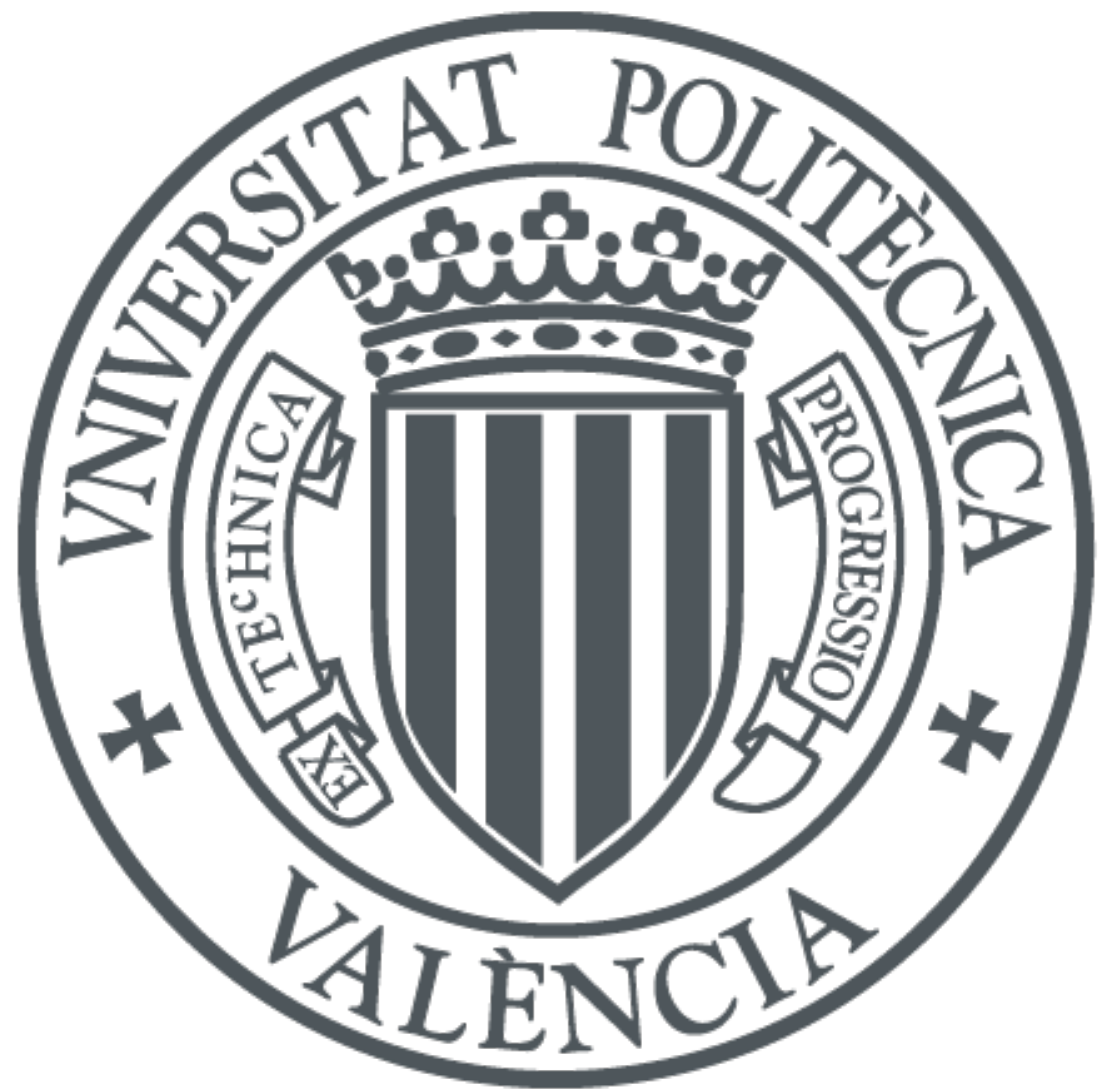

The final publication is available at

https://doi.org/10.1142/S0219530519500179

Copyright World Scientific

Additional Information 


\title{
WEIGHTED $p$-REGULAR KERNELS FOR REPRODUCING KERNEL HILBERT SPACES AND MERCER THEOREM
}

\author{
L. AGUD, J.M. CALABUIG* AND E. A. SÁNCHEZ PÉREZ**
}

\begin{abstract}
Let $(X, \Sigma, \mu)$ be a finite measure space and consider a Banach function space $Y(\mu)$. Motivated by some previous papers and current applications, we provide a general framework for representing reproducing kernel Hilbert spaces as subsets of Köthe-Bochner (vector valued) function spaces. We analyze operator-valued kernels $\Gamma$ that define integration maps $L_{\Gamma}$ between Köthe-Bochner spaces of Hilbert valued functions $Y(\mu ; \mathcal{K})$. We show a reduction procedure which allows to find a factorization of the corresponding kernel operator through weighted Bochner spaces $L^{p}(g d \mu ; \mathcal{K})$ and $L^{p^{\prime}}(h d \mu ; \mathcal{K})$-where $1 / p+1 / p^{\prime}=1$ - under the assumption of $p$-concavity of $Y(\mu)$. Equivalently, a new kernel obtained by multiplying $\Gamma$ by scalar functions can be given in such a way that the kernel operator is defined from $L^{p}(\mu ; \mathcal{K})$ to $L^{p^{\prime}}(\mu ; \mathcal{K})$ in a natural way. As an application, we prove a new version of Mercer Theorem for matrix valued weighted kernels.
\end{abstract}

\section{INTRODUCTION}

In recent years, some effort has been made for understanding representations of reproducing kernel Hilbert spaces (RKHSs) as subsets of Hilbert spaces of vector-valued functions. The motivation for this research line comes from several practical problems that are related to machine learning and multi-objective optimization, among other mathematical topics (see for example $[5,12,16,26]$ and the references therein). Indeed, recent developments in support vector machines and in general machine learning have shown that some advanced results in reproducing kernel Hilbert (Banach) spaces have found

2010 Mathematics Subject Classification. Primary 46E22; Secondary 46E30, 30C40, 47B34 .

Key words and phrases. Reproducing kernel Hilbert spaces; Integral operator; kernel operator; factorization; representation; Mercer Theorem.

* Support of the Ministerio de Economía y Competitividad (Spain), under project MTM2014-53009-P (Spain) is gratefully acknowledged.

** Support of the Ministerio de Ciencia, Innovación y Universidades (Spain), Agencia Estatal de Investigación, and FEDER under project MTM2016-77054-C2-1-P (Spain) is gratefully acknowledged. 
applications in these fields. Actually, the reader can find a relevant amount of papers of theoretical and applied nature in the current specialized literature on these areas (see for example $[7,13,21,24,25,27])$.

The primary setting for studying this kind of problems consists of an operator-valued kernel $\Gamma: X \times X \rightarrow L(\mathcal{K}, \mathcal{K})$ - where $\mathcal{K}$ is a Hilbert space- and the RKHS generated by $\Gamma$, which can be represented as a subspace of Hilbert space valued functions that belong to a Bochner space $L^{2}(\mu ; \mathcal{K})$. In the case that $\mathcal{K}$ is finite dimensional and adding the requirement of compactness of the associated kernel operator $L_{\Gamma}: L^{2}(\mu ; \mathcal{K}) \rightarrow L^{2}(\mu ; \mathcal{K})$, the Hilbert-Schmidt Theorem gives a spectral representation of $L_{\Gamma}$ that finally allows to obtain a Mercer decomposition of $\Gamma$, as in the scalar valued case. These results have been already presented in [11], where it is also shown how the metric requirements that are needed in the classical setting for proving Mercer Theorem can be relaxed.

A first extension of this framework to a bigger class of vector valued function spaces was given in [2], where vector valued RKHS are identified with subsets of $p$-Bochner integrable functions (see also [3]). The main result of this paper is a characterization of when such a RKHS, $\mathcal{H}_{\Gamma}$, for an operator valued kernel $\Gamma$ can be represented as a subset of a Bochner space $L^{p^{\prime}}(\mu ; \mathcal{K})$ - where $1 \leq p$ and $\mathcal{K}$ is a Hilbert space- It is given in terms of the associated kernel operator $L_{\Gamma}$, and establishes that $\mathcal{H}_{\Gamma} \subseteq L^{p}(\mu ; \mathcal{K})$ if and only if $L_{\Gamma}$ is is well defined as a weak integral from $L^{p^{\prime}}(\mu ; \mathcal{K})$ in $L^{p}(\mu ; \mathcal{K})$, where $1 / p+1 / p^{\prime}=1$. If this condition is satisfied the continuity of $L_{\Gamma}$ is ensured - see [2, Prop.4.4]-

The aim of the present paper is to show that these ideas can be generalized to a broader class of Köthe-Bochner spaces $Y(\mu ; \mathcal{K})$, including the ones generated by Lorentz-Bochner and Orlicz-Bochner spaces of Hilbert valued functions. Actually, Theorem 3.3 gives a generalization of Proposition 4.4 in [2], and constitutes one of our main results. Moreover, under the assumption of some lattice geometric properties for the space $Y(\mu)$, - mainly 2concavity - , we show a reduction procedure that allows to obtain a factorization through $L^{p^{\prime}}(\mu ; \mathcal{K})$ and $L^{p}(\mu ; \mathcal{K})$ of a weighted kernel operator $L_{\Gamma^{\prime}}$ associated to $\Gamma$ by the multiplication of two (scalar valued) weights. It is based on the so called Maurey-Rosenthal factorization theory for operators among Banach function spaces. The more relevant case 
- which is $p=2$ - is given in Theorem 3.4: under some Banach lattice assumptions on $Y(\mu)$ including 2-concavity, the inclusion $\mathcal{H}_{\Gamma} \subseteq Y(\mu ; \mathcal{K})$ implies a factorization through weighted Bochner spaces of vector valued 2-integrable functions, reducing the original setting to the known vector valued $L^{2}$-spaces framework. These results are presented in Section 3, after the Introduction (Section 1) and a preliminaries section (Section 2).

The results of Section 3 provide well-definition and continuity of $L_{\Gamma}$ through weighted Bochner $L^{p}$-spaces. However, two different weights appear in both extremes of the factorization scheme, which could make it difficult to describe the elements of $\mathcal{H}_{\Gamma}$ as vector valued functions. In fact, keep in mind that the spaces that appear in the definition of $L_{\Gamma}$ in the works we take as reference $($ see $[2,3])$ are always defined on the same measure of space. This motivates Section 4, where this problem is faced: a vector norm inequality for $L_{\Gamma}$ together with some convexity requirement for $Y(\mu)$ are provided as sufficient conditions for the kernel operator to be well-defined and continuous from $L^{p}(h d \mu ; \mathcal{K})$ in $L^{p^{\prime}}(h d \mu ; \mathcal{K})$ for a certain positive weight $h$ - the same $h$ in both sides of the operator $L_{\Gamma}$, see Proposition 4.2 - This result closes the analysis on the $p$-regularity of the kernel operators associated to vector valued RKHS.

The final Section 5 is devoted to show a concrete application of our circle of ideas for providing a weighted version of Mercer Theorem. Recall that Mercer Theorem gives a series representation for the reproducing (scalar) kernel that generates a given space. We are interested in describing RKHS that are represented by functions having values in a finite dimensional (complex) Euclidean space. This forces to consider matrix-valued kernels. In the classical case, the functions of the RKHS are scalar, $\mu$ is a finite measure (metric) space and we have that such a kernel defines a kernel operator $L_{\Gamma}: L^{2}(\mu) \rightarrow L^{2}(\mu)$. Typically, this provides a positive compact operator that gives an orthonormal basis $\left\{f_{i}\right\}_{i \in I}$ of eigenfunctions associated to a sequence of non-negative eigenvalues $\left\{\sigma_{i}\right\}_{i \in I}$ such that for $i \in I$ with $\sigma_{i}>0$, we have that $f_{i}$ is a continuous function. Using that, Mercer Theorem provides a representation of $\Gamma$ as

$$
\Gamma(x, y)=\sum_{j \in I} \sigma_{j} f_{j}(y) \overline{f_{j}(x)}, \quad x, y \in X
$$


For the case of matrix-valued kernels, a complete version of Mercer Theorem is given in [11]. In this paper, assumptions on the metric properties of the underlying measure space are removed, and a genuine Mercer Theorem for functions having values in a finite dimensional Hilbert space $\mathcal{K}$ is given. However, boundedness of the trace of the kernel and a change of measure is required for obtaining the desired representation by using that the associated kernel operator is defined, positive and compact as an endomorphism on $L^{2}(\nu ; \mathcal{K})$. In Section 5 we show that our factorization procedure allows to fix a similar result under different requirements - namely continuity of $L_{\Gamma}$ from the 2-concave lattice $Y(\mu ; \mathcal{K})$ to itself - to obtain another version of Mercer Theorem, that introduces some weights in the description of $\Gamma$ and in the basis of eigenfunctions of $L_{\Gamma}$ (see Theorem $5.4)$.

\section{StAndARD DEFINITIONS AND BASIC CONCEPTS}

Our notations on Banach spaces, integration and Banach function spaces is standard. As usual, if $E$ and $F$ are Banach spaces we write $L(E, F)$ for the space of linear bounded operators endowed with its natural norm. $E^{*}$ is the dual space of $E$, and $B_{E}$ is its closed unit ball.

Let us explain some notions related to Banach function spaces and $p$-th powers of spaces of integrable functions. We refer to $[19,20,22]$ for general questions related to Banach lattices, operators between them and spaces of integrable functions. Let $(X, \Sigma, \mu)$ be a $\sigma$-finite measure space. The space $L^{0}(\mu)$ is defined by all measurable real functions on $X$, where functions which are equal $\mu$-a.e. are considered to be the same. The $\mu$-a.e. pointwise order gives a lattice structure to this space. A Banach function space over $\mu$ is a Banach space $Y(\mu)$ of locally integrable functions in $L^{0}(\mu)([19, \mathrm{p} .28])$. All characteristic functions of measurable sets of finite measure are assumed to be in them, and they satisfy that if $|f| \leq|g|$ with $f \in L^{0}(\mu)$ and $g \in Y(\mu)$, then $f \in Y(\mu)$ and $\|f\| \leq\|g\|$.

Lattice properties of Banach function spaces will be relevant in this paper; if $Y(\mu)$ is so, it is said to be order continuous if every decreasing sequence $\left(f_{n}\right)_{n} \in Y(\mu)$ convergent to zero -i.e. $f_{n} \downarrow 0$-, satisfies that $\left\|f_{n}\right\|_{Y(\mu)} \rightarrow 0$. The set of all simple functions is 
dense in any order continuous Banach function space, and this is related to the properties of the so called Köthe dual. The Köthe dual $Y(\mu)^{\prime}$ of $Y(\mu)$ is the Banach function space over $\mu$ of all functions

$$
\left\{g \in L^{0}(\mu): f g \in L^{1}(\mu) \forall f \in Y(\mu)\right\}
$$

with the norm of the dual space,

$$
\|g\|_{Y(\mu)^{\prime}}:=\sup _{f \in B_{Y(\mu)}} \int_{X} f g d \mu
$$

It is known that a Banach function space is order continuous if and only if $Y(\mu)^{\prime}=Y(\mu)^{*}$ isometrically. A Banach function space $Y(\mu)$ has the Fatou property if every increasing sequence $\left(f_{n}\right)_{n}$ of non-negative functions in $Y(\mu)$ that is bounded in the norm, converges $\mu$-almost everywhere to a function $f$ belonging also to $Y(\mu)$ and satisfying $\sup _{n}\left\|f_{n}\right\|=$ $\|f\|$. A relevant characterization of this property is that the Köthe bidual $Y(\mu)^{\prime \prime}$ coincides with $Y(\mu)$ if and only if $Y(\mu)$ has the Fatou property ([19, p.29]). If $Y(\mu)$ and $Z(\mu)$ are Banach function spaces over the same measure $\mu$, we write $M(Y(\mu), Z(\mu))$ for the Banach function space of the functions $g$ that define operators, $M_{g}$, from $Y(\mu)$ to $Z(\mu)$ by means of the pointwise multiplication $M_{g}(f):=f \cdot g \in Z(\mu), f \in Y(\mu)$. Thus, the Köthe dual space is a particular case of space of multiplication operators. The norm $\|g\|_{M(Y(\mu), Z(\mu))}$ for a function $g$ in the space of multiplication operators is given by the operator norm of $M_{g},\left\|M_{g}\right\|$.

The computation of the pointwise power of functions belonging to the Banach function spaces allows to define a construction that will be fundamental in this paper. If $0<p<$ $\infty$, the $p$-th power of $Y(\mu)$ is the space of functions

$$
Y(\mu)_{[p]}:=\left\{f \in L^{0}(\mu):|f|^{1 / p} \in Y(\mu)\right\} .
$$

It is a Banach function space over $\mu$ with the norm

$$
\|f\|_{Y(\mu)_{[p]}}:=\left\||f|^{1 / p}\right\|_{Y(\mu)}^{p}, \quad f \in Y(\mu)_{[p]}
$$


whenever $Y(\mu)$ is $p$-convex (with $p$-convexity constant $M^{(p)}(Y(\mu))$ equal to 1 , see below). It is order continuous if and only if $Y(\mu)$ is so (see [22, Ch.2]). Recall that a Banach function space is $p$-convex if there is a constant $K>0$ such that for $f_{1}, \ldots, f_{n} \in Y(\mu)$,

$$
\left\|\left(\sum_{i=1}^{n}\left|f_{i}\right|^{p}\right)^{1 / p}\right\| \leq K\left(\sum_{i=1}^{n}\left\|f_{i}\right\|^{p}\right)^{1 / p}
$$

the infimum of the constants $K$ is the $p$-convexity constant $M^{(p)}(Y(\mu))$.

A Banach space valued operator $T: Y(\mu) \rightarrow E$ is said to be $p$-concave if there is a constant $K>0$ such that for $f_{1}, \ldots, f_{n} \in Y(\mu)$,

$$
\left(\sum_{i=1}^{n}\left\|T\left(f_{i}\right)\right\|^{p}\right)^{1 / p} \leq K\left\|\left(\sum_{i=1}^{n}\left|f_{i}\right|^{p}\right)^{1 / p}\right\| .
$$

The infimum of the constants $K$ is the $p$-concavity constant $M_{(p)}(T)$. A Banach function space is $p$-concave if the identity map $i d$ is $p$-concave.

Also, an operator $T: X \rightarrow Y$ between Banach lattices is called $p$-regular if it satisfies

$$
\left\|\left(\sum_{i=1}^{n}\left|T\left(f_{i}\right)\right|^{p}\right)^{1 / p}\right\| \leq K\left\|\left(\sum_{i=1}^{n}\left|f_{i}\right|^{p}\right)^{1 / p}\right\|,
$$

for all finite sets of elements $f_{1}, \ldots, f_{n} \in X$.

Let us introduce some notions on what are called Köthe-Bochner spaces of vector valued functions. The interested reader can find more information in the books $[4,18]$. If $Y(\mu)$ is a Banach function space and $E$ is a Banach space, the Köthe-Bochner space $Y(\mu ; E)$ is the space of $\mu$-a.e. equal classes of strongly measurable functions $f: X \rightarrow E$ satisfying that the function $\|f\|_{E}: X \rightarrow \mathbb{R}$ given by $\|f\|_{E}(x):=\|f(x)\|_{E}, x \in X$, belongs to $Y(\mu)$. The norm for this space is given by

$$
\|f\|_{Y(\mu ; E)}:=\|\| f\left\|_{E}(\cdot)\right\|_{Y(\mu)}, \quad f \in Y(\mu ; E)
$$

The resulting structure is a Banach space of (classes of) strongly measurable functions that are equal $\mu$-a.e. In particular, for $Y(\mu)=L^{p}(\mu)$ we obtain the classical Bochner spaces $L^{p}(\mu ; E)$. The Köthe duality — that is, the duality for elements of the topological dual that can be represented also as strongly integrable functions - is given (in the case 
of order continuous spaces) by

$$
\langle f, \phi\rangle:=\int_{X}\langle f(x), \phi(x)\rangle d \mu(x), \quad f \in Y(\mu ; E), \phi \in Y\left(\mu ; E^{*}\right)^{\prime} .
$$

We assume that in this case $Y(\mu ; E)^{*}=Y(\mu ; E)^{\prime}$, what happens for instance when $E$ is a Hilbert space. Actually, in the case of $E$ being a (complex) Hilbert space, the duality inside this integral should be substituted by the inner product. In general, in the Hilbert space case we adopt notation and concepts of the (complex) Hilbert space theory. For an operator $T$ between Hilbert spaces, we write $T^{*}$ for its adjoint operator. If $f$ is a complex function, $f^{*}$ is its (pointwise) conjugate function.

Finally let us report that the basic elements on Pettis integration that are needed can be found in $[15, \S .3 .7]$, for example. Pettis integration of vector functions is a vector valued Lebesgue-type integration; if $\Psi$ is a strongly measurable Pettis integrable function $\Psi: X \rightarrow E$, we denote by $w-\int_{X} \Psi d \mu \in E$ to its integral. It satisfies that

$$
\left\langle w-\int_{A} \Psi d \mu, x^{*}\right\rangle=\int_{A}\left\langle\Psi, x^{*}\right\rangle d \mu,
$$

for all $A \in \Sigma$ and $x^{*} \in E^{*}$.

\section{3. $Y(\mu)$-REgularity For VECTOR-VALUED REPRODUCing KERnel Hilbert SPACES}

Let $Y(\mu)$ be an order continuous Banach function lattice over $\mu$ and $Y(\mu)^{*}$ its dual space, that coincides with its Köthe dual $Y(\mu)^{\prime}$. Let us introduce some definitions and notations, and a brief explanation of how to construct the associated kernel for a reproducing kernel Hilbert space. We also assume that $\mathcal{K}$ is a separable Hilbert space.

Definition 3.1. Let $X$ be a set. We are assuming that $\mathcal{H}$ is a Hilbert space that can be identified with a space of strongly measurable functions $X \rightarrow \mathcal{K}$. Let $x \in X$. Consider the evaluation map $e v_{x}: \mathcal{H} \rightarrow \mathcal{K}$, that is given by $e v_{x}(f)=f(x), f \in \mathcal{H}$. As usual, we assume that all these operators are continuous, that is for all $x \in X$ there is a constant $C_{x}>0$ such that

$$
\|f(x)\|_{\mathcal{K}} \leq C_{x}\|f\|_{\mathcal{H}}, \quad f \in \mathcal{H}
$$


In this case, we say that $\mathcal{H}$ is a $\mathcal{K}$-valued reproducing kernel Hilbert space over $X(\mathcal{K}$ RKHS or simply RKHS for short).

Given such a space $\mathcal{H}$, we can construct the corresponding - operator valued - reproducing kernel associated to it as

$$
\Gamma: X \times X \rightarrow L(\mathcal{K}, \mathcal{K}), \quad \Gamma(x, y)=e v_{x} \circ e v_{y}^{*}, \quad x, y \in X
$$

Note that $\Gamma(x, y)^{*}=\Gamma(y, x)$ and

$$
\Gamma(x, y)(v)=\left(e v_{x} \circ e v_{y}^{*}\right)(v)=e v_{x}\left(e v_{y}^{*}(v)\right)=e v_{y}^{*}(v)(x),
$$

for each $x, y \in X$ and $v \in \mathcal{K}$. Therefore $\Gamma(\cdot, y)(v)=e v_{y}^{*}(v)$.

It must be understood that the kernel $\Gamma$ defined in this way allows to compute the value of $f(x)$ for all $f \in \mathcal{H}$ and $x \in X$, since

$$
\langle f(x), v\rangle_{\mathcal{K}}=\left\langle e v_{x}(f), v\right\rangle_{\mathcal{K}}=\left\langle f, e v_{x}^{*}(v)\right\rangle_{\mathcal{H}}=\langle f, \Gamma(\cdot, x)(v)\rangle_{\mathcal{H}}=\left\langle f, \Gamma(x, \cdot)^{*}(v)\right\rangle_{\mathcal{H}}
$$

For technical reasons, it is convenient to define the operator $\gamma: X \rightarrow L(\mathcal{K}, \mathcal{H})$ as the pointwise adjoint map

$$
\gamma(x)=e v_{x}^{*}: \mathcal{K} \rightarrow \mathcal{H}, \quad x \in X
$$

Thus, $\Gamma(x, y)=\gamma(x)^{*} \gamma(y)$ for all $x, y \in X$.

This construction can be made in a more abstract way by introducing an identification map instead of a natural inclusion (see Proposition 2.4 in [2]). Recall that a partial isometry between Hilbert spaces is a linear isometry on the orthogonal complement of its kernel. If $\widehat{\mathcal{H}}$ is Hilbert space that can be identified — not necessarily in an injective way - by a linear operator $A$ with a subspace of $\mathcal{K}^{X}$ — the linear space of functions from $X$ to the Hilbert space $\mathcal{K}$ - in such a way that

$$
\|A(u)(x)\|_{\mathcal{K}} \leq C_{x}\|u\|_{\widehat{\mathcal{H}}}, \quad x \in X, u \in \widehat{\mathcal{H}},
$$


then there is a reproducing kernel Hilbert space $\mathcal{H}$ such that there is a partial isometry from $\widehat{\mathcal{H}}$ on it, and with a reproducing kernel given by

$$
\Gamma(x, y):=\gamma(x)^{*} \gamma(y), \quad x, y \in X
$$

Here, $\gamma$ is defined as explained above starting from the reproducing kernel Hilbert space $\mathcal{H}$. The reader can find an explanation in Proposition 2.3 in [2]. This justifies the use of the representation of the corresponding kernel $\Gamma$ as $\gamma^{*} \gamma$, given a $\mathrm{RKH}$ space $\mathcal{H}$. It will be necessary for the proof of the following result.

The proof of the original theorem for $L^{p}$-spaces - the main result of [2], Proposition 4.4-, can be transferred to the following general case, as we show in what follows.

Definition 3.2. Let $\Gamma: X \times X \rightarrow L(\mathcal{K}, \mathcal{K})$ be a strongly measurable function. Given a Banach function space of $\mathcal{K}$-valued functions $Y(\mu ; \mathcal{K})$, we say that $\Gamma$ is $Y(\mu)$-bounded if

(i) for $\mu$-almost all $x \in X$ and $v \in B_{\mathcal{K}}$ we have that $\left\|\Gamma(x, \cdot)^{*}(v)\right\|_{\mathcal{K}} \in Y(\mu)^{\prime}$, and

(ii) for all $\phi \in Y(\mu ; \mathcal{K})$, the integration map

$$
x \rightsquigarrow w-\int_{X} \Gamma(x, y) \phi(y) d \mu(y)
$$

is in $Y(\mu ; \mathcal{K})^{\prime}$.

It is relevant to notice that thanks to (i) the expression appearing in (ii) is well defined; indeed, given $\phi \in Y(\mu ; \mathcal{K})$, we have that $y \rightsquigarrow\langle\Gamma(x, y) \phi(y), v\rangle_{\mathcal{K}}$ is measurable - for all $v \in \mathcal{K}$ - and we have the bound

$$
\begin{aligned}
\int_{X}\left|\langle\Gamma(x, y) \phi(y), v\rangle_{\mathcal{K}}\right| d \mu(y) & \leq \int_{X}\|\phi(y)\|_{\mathcal{K}}\left\|\Gamma(x, y)^{*}(v)\right\|_{\mathcal{K}} d \mu(y) \\
& =\int_{X}\|\phi\|_{\mathcal{K}}(y)\left\|\Gamma(x, \cdot)^{*}(v)\right\|_{\mathcal{K}}(y) d \mu(y) \\
& \leq\|\phi\|_{Y(\mu ; \mathcal{K})}\left\|\Gamma(x, \cdot)^{*}(v)\right\|_{Y(\mu ; \mathcal{K})^{\prime}} .
\end{aligned}
$$

This means that the strongly measurable function $\Gamma(x, \cdot) \phi$ is scalarly integrable - that is $\langle\Gamma(x, \cdot) \phi, v\rangle \in L^{1}(\mu)$ for all $v \in \mathcal{K}$ - and then - since $\mathcal{K}$ is supposed to be separable - the Pettis integral $w-\int_{X} \Gamma(x, y) \phi(y) d \mu(y)$ exists and belongs to $\mathcal{K}$ for $\mu$-almost all $x \in X$. 
Moreover, a classical Closed Graph Theorem argument shows easily that, if $\Gamma$ is $Y(\mu)$ bounded, then the operator $L_{\Gamma}$ defined by the Pettis integral of the kernel is continuous from $Y(\mu ; \mathcal{K})$ to $Y(\mu ; \mathcal{K})^{\prime}$ (see Proposition 4.2 in [2], taking into account that for a general Banach function space over a finite measure $\mu$ is also true that every norm convergent sequence has a subsequence that converges $\mu$-a.e.). For the factorization procedure that we will use in the rest of the paper, it is indeed relevant to notice that the operator

$$
L_{\Gamma}(\phi)(x):=w-\int_{X} \Gamma(x, y) \phi(y) d \mu(y), \quad \phi \in Y(\mu ; \mathcal{K}), x \in X,
$$

is continuous when defined as an operator $L_{\Gamma}: Y(\mu ; \mathcal{K}) \rightarrow Y(\mu ; \mathcal{K})^{\prime}$.

Theorem 3.3. Let $\mathcal{H}$ be a $R K H$ space of (strongly) measurable $\mathcal{K}$-valued functions. Let $Y(\mu)$ be an order continuous Banach function lattice with the Fatou property. The following conditions are equivalent.

(i) The elements of $\mathcal{H}$ belong to $Y(\mu ; \mathcal{K})$.

(ii) The reproducing kernel $\Gamma$ of $\mathcal{H}$ is $Y(\mu)^{\prime}$-bounded.

Proof. (i) $\Rightarrow$ (ii). A Closed Graph Theorem argument gives that the inclusion map $\iota_{\Gamma}: \mathcal{H} \rightarrow Y(\mu ; \mathcal{K})$ - that is well-defined by hypothesis- is also continuous - see for instance the proof of $[22$, Lemma 2.7] and the references therein-. Recall that $\Gamma$ - the associated kernel to the RKHS $\mathcal{H}$ - is directly related with the map $\gamma(x)$ introduced in the previous paragraphs, that is given by $\gamma(x)=e v_{x}^{*}$.

By the definition of the maps involved, it can be directly seen by transposing the involved operators that

$$
\begin{aligned}
\left\langle\iota_{\Gamma}^{*} \phi, f\right\rangle_{\mathcal{H}} & =\left\langle\phi, \iota_{\Gamma} f\right\rangle_{Y(\mu ; \mathcal{K})}=\int_{X}\langle\phi(x), f(x)\rangle_{\mathcal{K}} d \mu(x) \\
& =\int_{X}\left\langle\phi(x), e v_{x}(f)\right\rangle_{\mathcal{K}} d \mu(x)=\int_{X}\langle\gamma(x) \phi(x), f\rangle_{\mathcal{H}} d \mu(x),
\end{aligned}
$$

which gives that the function $\gamma(\cdot) \phi(\cdot)$ is weakly integrable and

$$
\iota_{\Gamma}^{*} \phi=w-\int_{X} \gamma(x) \phi(x) d \mu(x)
$$


Let us show that $\Gamma(x, y)=\gamma(x)^{*} \gamma(y)$ is a $Y(\mu)^{\prime}$-bounded kernel. If $x \in X$, then for $v \in \mathcal{K}$ recall that $\Gamma(x, \cdot)^{*}(v)=e v_{x}^{*}(v)$ is a function in $\mathcal{H}$ and so by assumption it belongs to $Y(\mu ; \mathcal{K})$. Since $Y(\mu)$ has the Fatou property, we have that the Köthe bidual $Y(\mu)^{\prime \prime}$ coincides with $Y(\mu)$, and so

$$
\left\|\Gamma(\cdot, y)^{*} v\right\|_{\mathcal{K}} \in\left(Y(\mu)^{\prime}\right)^{\prime}=Y(\mu), \quad v \in \mathcal{K}
$$

This gives (i) of Definition 3.2. On the other hand, if $\phi \in Y(\mu, \mathcal{K})^{\prime}$ then using Equation (1) and the properties of the Pettis integral we obtain that

$$
\begin{aligned}
w-\int_{X} \Gamma(x, y) \phi(y) d \mu(y) & =w-\int_{X} \gamma(x)^{*} \gamma(y) \phi(y) d \mu(y) \\
& =\gamma(x)^{*}\left(\int_{X} \gamma(y) \phi(y) d \mu(y)\right)=e v_{x}\left(\iota_{\Gamma}^{*} \phi\right)=\left(\iota_{\Gamma} \iota_{\Gamma}^{*} \phi\right)(x),
\end{aligned}
$$

for almost all $x \in X$. This gives (ii), since by definition $\iota_{\Gamma} \iota_{\Gamma}^{*}(\phi) \in Y(\mu ; \mathcal{K})$.

(ii) $\Rightarrow$ (i). Take a measurable partition $\left(A_{k}\right)_{k}$ of $X$ composed by sets of finite measure and write $C_{n}:=\left\{x \in \cup_{k=1}^{n} A_{k}:\|\gamma(x)\|_{\mathcal{B}(\mathcal{K}, \mathcal{H})} \leq n\right\}$. If $f \in \mathcal{H}$, define the functions $f_{n}:=\chi_{C_{n}} f$. Then we have that for every $x \in X$,

$$
\left\|f_{n}(x)\right\|_{\mathcal{K}}=\chi_{C_{n}}(x)\left\|e v_{x}(f)\right\|_{\mathcal{K}}=\chi_{C_{n}}(x)\left\|\gamma(x)^{*}(f)\right\|_{\mathcal{K}} \leq n \chi_{C_{n}}(x)\|f\|_{\mathcal{H}},
$$

and so $f_{n} \in Y(\mu ; \mathcal{K})$ since they are pointwise bounded and they have support of finite measure. This allows to compute the next duality

$$
\left\langle f_{n}, \phi\right\rangle_{Y(\mu ; \mathcal{K})}=\int_{X}\left\langle\chi_{C_{n}}(x) f(x), \phi(x)\right\rangle_{\mathcal{K}} d \mu(x)=\left\langle f, \int_{X} \chi_{C_{n}}(x) \gamma(x) \phi(x) d \mu(x)\right\rangle_{\mathcal{H}} .
$$


This allows to get the following bound, taking into account the properties of the Pettis integral of $\chi_{C_{n}}(y) \Gamma(x, y) \phi(y)$ which exists by hypothesis:

$$
\begin{aligned}
\left|\left\langle f_{n}, \phi\right\rangle_{Y(\mu ; \mathcal{K})}\right| & \leq\|f\|_{\mathcal{H}}\left\langle\int_{X} \chi_{C_{n}}(x) \gamma(x) \phi(x) d \mu(x), \int_{X} \chi_{C_{n}}(x) \gamma(x) \phi(x) d \mu(x)\right\rangle^{1 / 2} \\
& =\|f\|_{\mathcal{H}}\left\langle\int_{X} \chi_{C_{n}}(x) \gamma(x) \phi(x) d \mu(x), \int_{X} \chi_{C_{n}}(y) \gamma(y) \phi(y) d \mu(y)\right\rangle^{1 / 2} \\
& =\|f\|_{\mathcal{H}}\left(\int_{X} \int_{X}\left\langle\chi_{C_{n}}(x) \gamma(x) \phi(x), \chi_{C_{n}}(y) \gamma(y) \phi(y)\right\rangle_{\mathcal{H}} d \mu(x) d \mu(y)\right)^{1 / 2} \\
& =\|f\|_{\mathcal{H}}\left(\int_{X}\left\langle w-\int_{X} \chi_{C_{n}}(x) \Gamma(y, x) \phi(x) d \mu(x), \chi_{C_{n}}(y) \phi(y)\right\rangle_{\mathcal{K}} d \mu(y)\right)^{1 / 2} \\
& =\|f\|_{\mathcal{H}}\left(\left\langle L_{\Gamma}\left(\chi_{C_{n}} \phi\right), \chi_{C_{n}} \phi\right\rangle_{Y(\mu ; \mathcal{K})}\right)^{1 / 2} \leq\|f\|_{\mathcal{H}}\left\|L_{\Gamma}\right\|^{1 / 2}\|\phi\|_{Y(\mu, \mathcal{K})^{\prime}},
\end{aligned}
$$

where the equality $\Gamma(x, y)^{*}=\Gamma(y, x)$ has been used. The Fatou property of $Y(\mu)$ implies that the subspace $Y(\mu)^{\prime}$ of the topological dual $Y(\mu)^{*}$ is norming for $Y(\mu)$ by Proposition 1.b.18 [19]. That is, for every $h \in Y(\mu)$,

$$
\|h\|_{Y(\mu)}=\sup _{z \in B_{Y(\mu)^{\prime}}}\left|\int_{X} h(x) z(x) d \mu(x)\right|
$$

Now we claim that this property is transferred to the corresponding Köthe-Bochner spaces of Hilbert space valued integrable functions. Indeed, let $g \in Y(\mu)^{\prime}$ be an almost norming (up to an $\varepsilon$ ) norm one real function. Now, for $x \rightsquigarrow\|f(x)\|_{\mathcal{K}}$ consider the function $\widehat{g}(x):=g(x) \cdot f^{*}(x) /\|f(x)\|_{\mathcal{K}}$. It clearly belongs to $Y(\mu ; \mathcal{K})^{\prime}$ since it is strongly measurable and

$$
\|\| \widehat{g}(x)\left\|_{\mathcal{K}}\right\|_{Y(\mu)^{\prime}}=\|g(x)\|_{Y(\mu)^{\prime}} .
$$

On the other hand,

$$
\begin{aligned}
\left|\langle f, \widehat{g}\rangle_{Y(\mu ; \mathcal{K})}\right| & =\left|\int_{X}\langle f(x), \widehat{g}(x)\rangle_{\mathcal{K}} d \mu(x)\right| \\
& =\left|\int_{X}\left\langle f(x), g(x) \frac{f^{*}(x)}{\|f(x)\|_{\mathcal{K}}}\right\rangle_{\mathcal{K}} d \mu(x)\right| \\
& =\left|\int_{X}\|f(x)\|_{\mathcal{K}} g(x) d \mu(x)\right|>\|\| f(x)\left\|_{\mathcal{K}}\right\|_{Y(\mu)}-\varepsilon=\|f\|_{Y(\mu ; \mathcal{K})}-\varepsilon
\end{aligned}
$$


Since this holds for every $\varepsilon>0$ we have proven the claim.

Using this observation and the previous inequalities, we get

$$
\begin{aligned}
\left\|f_{n}\right\|_{Y(\mu ; \mathcal{K})} & =\sup _{\phi \in B_{Y(\mu ; \mathcal{K})^{\prime}}}\left|\left\langle f_{n}, \phi\right\rangle\right| \\
& =\sup _{\phi \in B_{Y(\mu ; \mathcal{K})^{\prime}}}\left|\int_{X}\left\langle f_{n}(x), \phi(x)\right\rangle_{\mathcal{K}} d \mu(x)\right| \leq\left\|L_{\Gamma}\right\|^{1 / 2}\|f\|_{\mathcal{H}} .
\end{aligned}
$$

Now we use again the Fatou property for $Y(\mu)$. We have an increasing sequence of functions $\left(g_{n}\right)$ defined by $g_{n}(x)=\left\|f_{n}(x)\right\|_{\mathcal{K}}$ in the space $Y(\mu)$ converging pointwise to the measurable function $x \rightsquigarrow\|f(x)\|_{\mathcal{K}}$ such that

$$
\sup _{n}\left\|g_{n}\right\|_{Y(\mu)}=\sup _{n}\|\| f_{n}(x)\left\|_{\mathcal{K}}\right\|_{Y(\mu)} \leq\left\|L_{\Gamma}\right\|^{1 / 2}\|f\|_{\mathcal{H}}
$$

Then $\|f(\cdot)\|_{\mathcal{K}} \in Y(\mu)$, and so $f \in Y(\mu ; \mathcal{K})$ with $\sup _{n}\left\|g_{n}\right\|_{Y(\mu)}=\|f\|_{Y(\mu ; \mathcal{K})}$.

Therefore when $\Gamma$ is $Y(\mu)^{\prime}$-bounded - or, equivalently when $\mathcal{H}$ is a subspace of $Y(\mu ; \mathcal{K})$ - then

$$
L_{\Gamma}=\iota_{\Gamma} \iota_{\Gamma}^{*}: Y(\mu ; \mathcal{K})^{\prime} \rightarrow Y(\mu ; \mathcal{K})
$$

is well defined and continuous.

A direct application of some classical Maurey-Rosenthal type factorization arguments provides the following result, that implies a certain weighted 2-regularity for the kernel operator $L_{\Gamma}$. For the case of $L^{p}(\mu ; \mathcal{K})$ spaces, with $\mu$ finite, the next result is obvious: indeed, if we consider $Y(\mu)=L^{p}(\mu)$ and taking into account that $L^{p}(\mu)$ is 2-convex whenever $2 \leq p$, we get the inclusions

$$
\mathcal{H} \subseteq Y(\mu ; \mathcal{K})=L^{p}(\mu ; \mathcal{K}) \subseteq L^{2}(\mu ; \mathcal{K})
$$

We prove in what follows that for this inclusion to be true - up to a multiplicative weight-, is enough $Y(\mu)^{\prime}$ to be 2-convex - i.e. $Y(\mu)$ is 2-concave, [19, Prop.1.d.4.(i)] -

Theorem 3.4. Let $\mathcal{H}$ be a $R K H$ space of (strongly) measurable $\mathcal{K}$-valued functions. Let $Y(\mu)$ be a 2-concave Banach function space with the Fatou property and such that $Y(\mu)^{\prime}$ 
is order continuous, and suppose that

$$
\mathcal{H} \subseteq Y(\mu ; \mathcal{K})
$$

Then there are weights $f \in M\left(Y(\mu)^{\prime}, L^{2}(\mu)\right)$ and $g \in M\left(L^{2}(\mu), Y(\mu)\right)$ such that the kernel $\Gamma$ associated to $\mathcal{H}$ defines a continuous operator

$$
L_{\Gamma}: L^{2}\left(f^{2} d \mu ; \mathcal{K}\right) \rightarrow L^{2}\left(g^{-2} d \mu ; \mathcal{K}\right)
$$

and $\mathcal{H} \subseteq L^{2}\left(f^{2} d \mu ; \mathcal{K}\right)$

Proof. By Theorem 3.3, we have that the kernel operator is $Y(\mu)^{\prime}$-bounded, and so the corresponding kernel operator $L_{\Gamma}$ defined by the Pettis integral

$$
L_{\Gamma}(\phi)(x)=w-\int_{X} \Gamma(x, y) \phi(y) d \mu(y),
$$

is continuous from $Y(\mu ; \mathcal{K})^{\prime}$ to $Y(\mu ; \mathcal{K})^{\prime \prime}$. Then the operator is defined from $Y(\mu ; \mathcal{K})^{\prime}$ to $Y(\mu ; \mathcal{K})$, since $Y(\mu)$ has the Fatou property. Thus, the range of $L_{\Gamma}$ is $Y(\mu ; \mathcal{K})$. Under the assumption of 2-concavity of $Y(\mu)$, it is known that $Y(\mu)^{\prime}$ is 2-convex - see [19, Prop.1.d.4.(i)] - Also, each operator between Banach lattices is 2-regular by Krivine's version of Grothendieck Theorem — see Theorem 1.f.14 in [19], [17]—.

We can now apply a separation argument - a Maurey-Rosenthal factorization - for getting a continuous operator between weighted $L^{2}$-spaces of vector valued functions. The result can be obtained just by applying Theorem 3.1 in [10], for $s=r=2$. It gives the existence of functions $f \in M\left(Y(\mu)^{\prime}, L^{2}(\mu)\right)$ and $g \in M\left(L^{2}(\mu), Y(\mu)\right)$ that provide a factorization for $L_{\Gamma}$ as

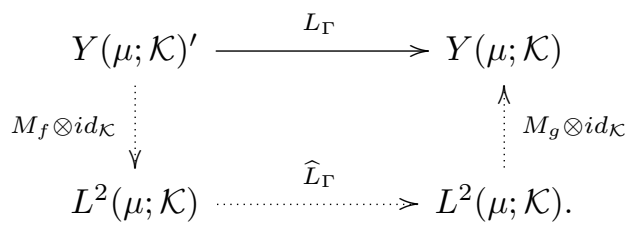


Using this factorization diagram, we get that $L_{\Gamma}(\phi)=g \widehat{L}_{\Gamma}(f \phi)$, for every $\phi \in Y(\mu ; \mathcal{K})^{\prime}$. Moreover, since $\widehat{L}_{\Gamma}$ is continuous from $L^{2}(\mu ; \mathcal{K})$ to $L^{2}(\mu ; \mathcal{K})$, we have that:

$$
\begin{aligned}
\left\|L_{\Gamma}(\phi)\right\|_{L^{2}\left(g^{-2} d \mu, \mathcal{K}\right)}^{2} & =\int_{X}\left\|L_{\Gamma}(\phi)(x)\right\|_{\mathcal{K}}^{2} \frac{d \mu(x)}{g(x)^{2}}=\int_{X}\left\|\widehat{L}_{\Gamma}(f \phi)(x)\right\|_{\mathcal{K}}^{2} d \mu(x) \\
& \leq\left\|\widehat{L}_{\Gamma}\right\|^{2} \int_{X}\|\phi(x)\|_{\mathcal{K}}^{2} f(x)^{2} d \mu(x)=\left\|\widehat{L}_{\Gamma}\right\|^{2}\|\phi\|_{L^{2}\left(f^{2} d \mu, \mathcal{K}\right)}
\end{aligned}
$$

Therefore, the operator $L_{\Gamma}$ is defined and continuous from $L^{2}\left(f^{2} d \mu ; \mathcal{K}\right)$ to $L^{2}\left(g^{-2} d \mu ; \mathcal{K}\right)$. Clearly, we also have that $\mathcal{H} \subseteq L^{2}\left(f^{2} d \mu ; \mathcal{K}\right)$.

Remark 3.5. A similar result than the one given in Theorem 3.4 can be obtained for an index $p \leq 2$ using the same factorization argument and assuming $p$-concavity for $Y(\mu)$. In this case we directly get a factorization through weighted Bochner $L^{p}$-spaces. Indeed, if $Y(\mu)$ is a $p$-concave Banach function space with the Fatou property and such that $Y(\mu)^{\prime}$ is order continuous, and $\mathcal{H} \subseteq Y(\mu ; \mathcal{K})$, then there are weights $f \in M\left(Y(\mu)^{\prime}, L^{p}(\mu)\right)$ and $g \in M\left(L^{p}(\mu), Y(\mu)\right)$ such that the kernel $\Gamma$ associated to $\mathcal{H}$ defines a continuous operator

$$
L_{\Gamma}: L^{p}\left(f^{p} d \mu ; \mathcal{K}\right) \rightarrow L^{p}\left(g^{-p} d \mu ; \mathcal{K}\right)
$$

and $\mathcal{H} \subseteq L^{p}\left(f^{p} d \mu ; \mathcal{K}\right)$. In particular, since $p^{\prime} \geq p$ we have that $L^{p^{\prime}}\left(f^{p} d \mu ; \mathcal{K}\right) \subseteq L^{p}\left(f^{p} d \mu ; \mathcal{K}\right)$, and then we get that $L_{\Gamma}$ is well defined and continuous from $L^{p^{\prime}}\left(f^{p} d \mu ; \mathcal{K}\right)$ to $L^{p}\left(g^{-2} d \mu ; \mathcal{K}\right)$.

\section{Weighted $p$-REgularity For VeCtor-VAlued Reproducing Kernel Hilbert} SPACES

In this section we will show a sufficient condition of when a given kernel provides a RKHS that is included in some weighted $L^{p}$-space, for a weight belonging to a particular Banach function lattice. The main difference with the results of the previous section is that the weights appearing in both factorization spaces are in fact the same weight. Our idea is to use some factorization technique for linear operators in order to give a vector norm inequality that characterizes when a given kernel produces a RKHS being $p$-regular for some given $1 \leq p<\infty$. Recall that the operator $L_{\Gamma}$ is well-defined and continuous when defined between some Banach function spaces, typically $L_{\Gamma}: Y(\mu ; \mathcal{K}) \rightarrow Y(\mu ; \mathcal{K})^{\prime}$. 
This is the case, for instance, when $\Gamma$ is $Y(\mu)$-bounded being $Y(\mu)$ order continuous and having the Fatou property.

Assume also that $Y(\mu)$ is $p / 2$-convex. Consider the inequality

$$
\left(\sum_{i=1}^{n}\left\|\left\langle L_{\Gamma}\left(f_{i}\right), \phi_{i}\right\rangle\right\|_{Y(\mu)_{[p / 2]}}^{p / 2}\right)^{2 / p} \leq K\left\|\left(\sum_{i=1}^{n}\left\|f_{i}\right\|^{p / 2}\left\|\phi_{i}\right\|^{p / 2}\right)^{2 / p}\right\|_{Y(\mu)}
$$

for $f_{1}, \ldots, f_{n} \in Y(\mu ; \mathcal{K}), \phi_{1}, \ldots, \phi_{n} \in Y(\mu ; \mathcal{K})^{\prime}$. Note that the right hand expression in this formula is not necessarily defined, since $\left\|f_{i}\right\|\left\|\phi_{i}\right\| \in L^{1}(\mu)$, but in general $L^{1}(\mu)$ is not included in $Y(\mu)$. We will assume that the value of this part equals $\infty$ in this case.

Example 4.1. Let us show the canonical case that inspires Proposition 4.2. In this example, both statements in this proposition are equivalent. For $p=2$ and $Y(\mu)=L^{1}(\mu)$ we get that $Y(\mu)_{[p / 2]}=Y(\mu)_{[1]}=L^{1}(\mu)$, and so, by the 1-concavity of $L^{1}(\mu)$ - with constant 1 - we get

$$
\begin{aligned}
\left(\sum_{i=1}^{n}\left\|\left\langle L_{\Gamma}\left(f_{i}\right)(w), \phi_{i}(w)\right\rangle\right\|_{Y(\mu)_{[p / 2]}}^{p / 2}\right)^{2 / p} & =\sum_{i=1}^{n}\left\|\left\langle L_{\Gamma}\left(f_{i}\right)(w), \phi_{i}(w)\right\rangle\right\|_{L^{1}(\mu)} \\
& \leq\left\|\sum_{i=1}^{n}\right\| f_{i}(w)\|\| \phi_{i}(w)\|\|_{L^{1}(\mu)} \\
& =\left\|\left(\sum_{i=1}^{n}\left\|f_{i}\right\|^{p / 2}\left\|\phi_{i}\right\|^{p / 2}\right)^{2 / p}\right\|_{Y(\mu)},
\end{aligned}
$$

for $f_{1}, \ldots, f_{n} \in Y(\mu ; \mathcal{K}), \phi_{1}, \ldots, \phi_{n} \in Y(\mu ; \mathcal{K})^{\prime}$.

Next result shows that Inequality (2) implies that the kernel operator $L_{\Gamma}$ is well defined when considered as an operator between $L^{p}\left(h_{0} d \mu\right)$ and $L^{p^{\prime}}\left(h_{0} d \mu\right)$ for some weight $h_{0}$. We prove it for a general operator $T$ although it will be used for $T$ being $L_{\Gamma}$.

Proposition 4.2. Let $p \geq 2$. Suppose that the measure $\mu$ is finite. Consider the kernel $\Gamma$ and assume that $Y(\mu)$ is order continuous, $p / 2$-convex with $p / 2$-convexity constant equal to one. Suppose that $T$ is defined as an operator $T: Y(\mu ; \mathcal{K}) \rightarrow Y(\mu, \mathcal{K})^{\prime}$, and that there is a constant $K>0$ such that the inequality

$$
\left(\sum_{i=1}^{n}\left\|\left\langle T\left(f_{i}\right)(w), \phi_{i}(w)\right\rangle\right\|_{Y(\mu)_{[p / 2]} / 2}^{p}\right)^{2 / p} \leq K\left\|\left(\sum_{i=1}^{n}\left\|f_{i}(w)\right\|^{p / 2}\left\|\phi_{i}(w)\right\|^{p / 2}\right)^{2 / p}\right\|_{Y(\mu)},
$$


holds for $f_{1}, \ldots, f_{n} \in Y(\mu ; \mathcal{K}), \phi_{1}, \ldots, \phi_{n} \in Y(\mu ; \mathcal{K})^{\prime}$. Then there is a weight function

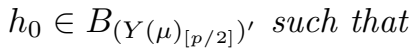

$$
\|T(f)\|_{L^{p^{\prime}\left(h_{0} d \mu ; \mathcal{K}\right)}} \leq K\|f\|_{L^{p}\left(h_{0} d \mu ; \mathcal{K}\right)}, \quad f \in Y(\mu ; \mathcal{K})
$$

Consequently, there is a factorization for $T$ as

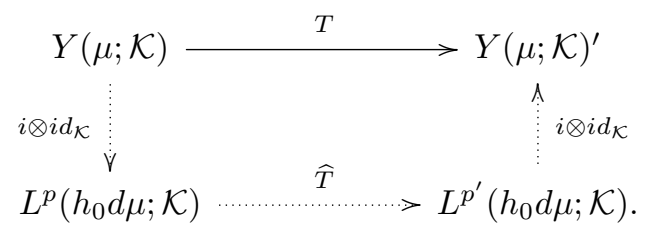

Proof. First note that $Y(\mu)_{[p / 2]}$ is a Banach space and the canonical quasinorm defined in the power is actually a norm, due to the fact that the $p / 2$-convexity constant is equal to one. Since $Y(\mu)$ is order continuous, we have that the $p / 2$-th power is order continuous too — see [22, Lemma 2.21(iii)] —, and so $Y(\mu)^{*}=Y(\mu)^{\prime}$. We assume that $1<p / 2$ - the proof for the case $p=2$ follows exactly the same lines-. Consider $f_{1}, \ldots, f_{n} \in Y(\mu ; \mathcal{K})$ and $\phi_{1}, \ldots, \phi_{n} \in Y(\mu ; \mathcal{K})^{\prime}$ such that the (class of $\mu$-a.e equal) function(s)

$$
w \mapsto\left\|f_{i}(w)\right\|_{\mathcal{K}}\left\|\phi_{i}(w)\right\|_{\mathcal{K}},
$$

belong(s) to $Y(\mu)$, for $i=1, \ldots, n$. Note that in general these functions are in $L^{1}(\mu)$, but we need to consider the case when they are in $Y(\mu)$ for the inequality (2) to make sense.

Note that the expression

$$
\left\|\left(\sum_{i=1}^{n}\left\|f_{i}\right\|^{p / 2}\left\|\phi_{i}\right\|^{p / 2}\right)^{2 / p}\right\|_{Y(\mu)},
$$

can be also written as

$$
\left\|\sum_{i=1}^{n}\right\| f_{i}\left\|^{p / 2}\right\| \phi_{i}\left\|^{p / 2}\right\|_{Y(\mu)_{[p / 2]}}^{2 / p},
$$

that is, the supremum of all the numbers

$$
\left(\int_{X} \sum_{i=1}^{n}\left\|f_{i}\right\|^{p / 2}\left\|\phi_{i}\right\|^{p / 2} h d \mu\right)^{2 / p},
$$


for $h \in B_{\left(Y(\mu)_{[p / 2]}\right)^{\prime}}$. Consider the set of functions $\Psi: B_{\left(Y(\mu)_{[p / 2]}\right)^{\prime}} \rightarrow \mathbb{R}$ defined as

$$
\Psi(h):=\sum_{i=1}^{n}\left\|\left\langle T\left(f_{i}\right), \phi_{i}\right\rangle\right\|_{Y(\mu)_{[p / 2]} / 2}^{p}-K^{p / 2} \sum_{i=1}^{n} \int_{X}\left\|f_{i}\right\|^{p / 2}\left\|\phi_{i}\right\|^{p / 2} h d \mu,
$$

for families of functions that satisfy that $\left\|f_{i}\right\|\left\|\phi_{i}\right\| \in Y(\mu)$ for $i=1, \ldots, n$. It is relevant to remark that simple functions are dense in $Y(\mu ; \mathcal{K})$ as a consequence of the order continuity of $Y(\mu)$, and couples $s, \phi$ defined for a simple function $s$ and any other function $\phi \in Y(\mu ; \mathcal{K})^{\prime}$ give functions $w \mapsto\|s(w)\|\|\phi(w)\|$ belonging to $Y(\mu)^{\prime}$. Notice that these functions are weak*-continuous, convex, and each convex combination of a finite set of these functions is again a member of the family. We can use a standard Ky Fan separation argument - the same that provides the Maurey-Rosenthal factorization of operators, see for example Section 3 in [1], Theorem 3.2 in this paper or Theorem 2 in [8]-, for obtaining a single function $h_{0} \in B_{\left(Y(\mu)_{[p / 2]}\right)^{\prime}}$ such that for each pair of functions $f \in Y(\mu ; \mathcal{K})$ and $\phi \in Y(\mu ; \mathcal{K})^{\prime}$ - for $f$ being simple - we have

$$
\|\langle T(f), \phi\rangle\|_{Y(\mu)_{[p / 2]} / 2}^{p / 2} \leq K^{p / 2} \int_{X}\|f\|^{p / 2}\|\phi\|^{p / 2} h_{0} d \mu .
$$

This implies that

$$
\begin{aligned}
\left(\int_{X}|\langle T(f), \phi\rangle| h_{0} d \mu\right)^{p / 2} & \leq\|\langle T(f), \phi\rangle\|_{Y(\mu)_{[p / 2]}^{p / 2}}^{p} \\
& \leq K^{p / 2} \int_{X}\|f\|^{p / 2}\|\phi\|^{p / 2} h_{0} d \mu \\
& \leq K^{p / 2}\left(\int_{X}\|f\|^{p} h_{0} d \mu\right)^{1 / 2}\left(\int_{X}\|\phi\|^{p} h_{0} d \mu\right)^{1 / 2},
\end{aligned}
$$

and so

$$
\int_{X}|\langle T(f), \phi\rangle| h_{0} d \mu \leq K\left(\int_{X}\|f\|^{p} h_{0} d \mu\right)^{1 / p}\left(\int_{X}\|\phi\|^{p} h_{0} d \mu\right)^{1 / p} .
$$

Therefore, a "duality computation" — taking into account that simple functions are dense in $L^{p}\left(h_{0} d \mu ; \mathcal{K}\right)$ - gives

$$
\|T(f)\|_{L^{p^{\prime}}\left(h_{0} d \mu ; \mathcal{K}\right)}=\sup _{\phi \in B_{L^{p}\left(h_{0} d \mu ; \mathcal{K}\right)}} \int_{X}|\langle T(f), \phi\rangle| h_{0} d \mu \leq K\left(\int_{X}\|f\|^{p} h_{0} d \mu\right)^{1 / p} .
$$


This implies that $T$ is a bounded operator when defined from $L^{p}\left(h_{0} d \mu ; \mathcal{K}\right)$ to $L^{p^{\prime}}\left(h_{0} d \mu ; \mathcal{K}\right)$; for general functions $f \in L^{p}\left(h_{0} d \mu ; \mathcal{K}\right)$, we use that simple functions are dense in the space.

Finally, density of simple functions in both $Y(\mu ; \mathcal{K})$ and $L^{p}\left(h_{0} d \mu ; \mathcal{K}\right)$ gives the factorization.

Remark 4.3. There are some relevant facts concerning the previous results that should be noticed. Let us explain them below.

(i) If we assume that there is a constant $K^{\prime}>0$ such that $\left\|L_{\Gamma}(f)(w)\right\|_{\mathcal{K}} \leq K^{\prime}\|f(w)\|_{\mathcal{K}}$ $\mu$-a.e. for all $f$, we can prove a sort of converse of the previous result. That is, under this requirement if we have a factorization for $L_{\Gamma}$ as the one provided in Proposition 4.2, then Inequality (2) holds for $Y(\mu)=L^{p / 2}\left(h_{0} d \mu\right)$. Indeed, taking $Y(\mu)=L^{p / 2}\left(h_{0} d \mu\right)$ then $Y(\mu)_{[p / 2]}=\left(L^{p / 2}\left(h_{0} d \mu\right)\right)_{[p / 2]}=L^{1}\left(h_{0} d \mu\right)$. Fix $f_{1}, \ldots, f_{n} \in L^{p / 2}\left(h_{0} d \mu ; \mathcal{K}\right), \phi_{1}, \ldots, \phi_{n} \in L^{(p / 2)^{\prime}}\left(h_{0} d \mu ; \mathcal{K}\right)$. Then

$$
\begin{gathered}
\left(\sum_{i=1}^{n}\left\|\left\langle L_{\Gamma}\left(f_{i}\right), \phi_{i}\right\rangle\right\|_{\left(L^{p / 2}\left(h_{0} d \mu\right)\right)_{[p / 2]}}^{p / 2}\right)^{2 / p} \\
=\left(\sum_{i=1}^{n}\left|\int_{X}\right|\left\langle L_{\Gamma}\left(f_{i}\right)(w), \phi_{i}(w)\right\rangle\left|h_{0}(w) d \mu(w)\right|^{p / 2}\right)^{2 / p} \\
\leq\left(\sum_{i=1}^{n}\left|\int_{X}\left\|L_{\Gamma}\left(f_{i}\right)(w)\right\|\left\|\phi_{i}\right\|(w) h_{0}(w) d \mu\right|^{p / 2}\right)^{2 / p} \\
\leq K\left(\sum_{i=1}^{n}\left|\int_{X}\left\|f_{i}(w)\right\|\left\|\phi_{i}\right\|(w) h_{0}(w) d \mu\right|^{p / 2}\right)^{2 / p} .
\end{gathered}
$$

Note that, since we are assuming that $\mu$ is finite measure, we have that the constant $Q=K\left(\int_{X} h_{0} d \mu\right)^{1 /(p / 2)^{\prime}}$ is finite. Since the space $L^{1}\left(h_{0} d \mu\right)$ is $r$ concave with constant 1 for all $r \geq 1$ and we assume that there is $K>0$ such 
that $\left\|L_{\Gamma}(f)(w)\right\|_{\mathcal{K}} \leq K\|f(w)\|_{\mathcal{K}}$ for all $f$, we get

$$
\begin{aligned}
(3) & \leq K \int_{X}\left(\sum_{i=1}^{n}\left\|f_{i}\right\|^{p / 2}\left\|\phi_{i}\right\|^{p / 2}\right)^{2 / p} h_{0} d \mu \\
& \leq K\left(\int_{X} \sum_{i=1}^{n}\left\|f_{i}\right\|^{p / 2}\left\|\phi_{i}\right\|^{p / 2} h_{0} d \mu\right)^{2 / p} \cdot\left(\int_{X} h_{0} d \mu\right)^{1 /(p / 2)^{\prime}} \\
& =Q\left\|\left(\sum_{i=1}^{n}\left\|f_{i}\right\|^{p / 2}\left\|\phi_{i}\right\|^{p / 2}\right)^{2 / p}\right\|_{L^{p / 2}\left(h_{0} d \mu\right)}
\end{aligned}
$$

So we obtain the required inequality.

(ii) We may also prove that there is a constant $K^{\prime}>0$ such that

$$
\int_{A}\left\|L_{\Gamma}(f)(w)\right\|_{\mathcal{K}} d \mu \leq K^{\prime}\|\| f(w)\left\|_{\mathcal{K}} \chi_{A}\right\|_{Y(\mu)}
$$

for every $f$ and $A \in \Sigma$, that is, the operator $L_{\Gamma}$ is defined as an operator $L_{\Gamma}$ : $Y(\mu ; \mathcal{K}) \rightarrow L^{1}(\mu ; \mathcal{K})$. Indeed, consider the functions $\phi$ of the dual space defined $\mu$-a.e. as $\phi(w)=\left(L_{\Gamma}(f)(w) /\left\|L_{\Gamma}(f)(w)\right\|\right) \chi_{A}$, for $A \in \Sigma$ and $f$ in the domain space. Recall that by the $p / 2$-convexity of $Y(\mu)$ we have that its $p / 2$-th power is a Banach function space, and so $Y(\mu)_{[p / 2]} \subseteq L^{1}(\mu)$, what gives the existence of a constant $c>0$ such that $c\|\cdot\|_{L^{1}(\mu)} \leq\|\cdot\|_{Y}(\mu)_{[p / 2]}$. Then Inequality (2) gives for a fixed function $f$ and such a $\phi$,

$c \int_{A}\left\|L_{\Gamma}(f)\right\|_{\mathcal{K}} d \mu \leq\left\|\left\langle L_{\Gamma}(f), \frac{L_{\Gamma}(f)}{\left\|L_{\Gamma}(f)\right\|} \chi_{A}\right\rangle\right\|_{Y(\mu)_{[p / 2]}} \leq K\|\| f \|_{\mathcal{K} \chi_{A} \|_{Y(\mu)}}$.

\section{Applications: Weighted norm inequalities for Kernels and Mercer}

\section{THEOREM}

Inspired in the results of [11], in this section we center our attention in the case of spaces of functions having values in finite dimensional (complex) Hilbert spaces. The main reason is that in this case it is possible to find interesting versions of Mercer Theorem that allows to compute the elements of the kernel representation and the related orthonormal basis by means of some explicit formulas. We will show a weak version of Mercer Theorem, in which the requirements for the original kernel are given by some 
norm inequalities for the associated kernel operator that are weaker than the usual requirements. The spaces of functions involved are in this case $\mathbb{C}^{n}$-valued. Recall that a space $L^{2}\left(\mu ; \mathbb{C}^{n}\right)$ can be considered as a Hilbert space by using the inner product given by the $\mathbb{C}^{n}$-inner product of the corresponding coordinate functions.

Let us introduce the setting for the finite dimensional case. We fix $\mathcal{K}$ to be the Euclidean space $\mathbb{C}^{n}$. First, it must be noticed that in this case the kernel is given by a measurable function $\Gamma: X \times X \rightarrow L\left(\mathbb{C}^{n}, \mathbb{C}^{n}\right)=\mathbb{M}_{n \times n}(\mathbb{C})$, where $\mathbb{M}_{n \times n}(\mathbb{C})$ is the space of complex valued $n \times n$-matrices endowed with the operator norm.

In this case, a "coordinate-based" decomposition method allows to use the results given by the scalar version of Mercer Theorem in a uniform way for providing the desired result. The duality relation is given by the inner product of the complex Euclidean space. Suppose that $\Gamma: X \times X \rightarrow \mathbb{M}_{n \times n}(\mathbb{C})$ is a $\mathbb{M}_{n \times n}(\mathbb{C})$-reproducing kernel, that is (see [11, Definition 2.1]): 1) for all $x, y \in X$ we have $\Gamma(x, y)^{*}=\Gamma(y, x)$, and 2) the following positivity assumption: for all $m, x_{1}, \ldots, x_{m} \in X$ and $y_{1}, \ldots, y_{m} \in \mathbb{C}^{n}$,

$$
\sum_{i, j=1}^{n}\left\langle\Gamma\left(x_{i}, x_{j}\right) y_{j}, y_{i}\right\rangle \geq 0
$$

In all the section $\mu$ is a Borel measure on a topological space $X$ and that the support of the measure $\mu$ is all the set $X$. For obtaining a Mercer decomposition of the kernel $\Gamma$ the usual requirements are centered in proving that the kernel operator $L_{\Gamma}$ is well-defined, positive and compact. Under these requirements, it is possible to use the Hilbert-Schmidt Theorem to the kernel operator for obtaining a basis of $L^{2}\left(\mu ; \mathbb{C}^{n}\right)$ that gives the key for getting the Mercer decomposition of $\Gamma$.

In [11, Th.3.4], this is obtained by changing the measure on the space and asking for the integrability of the trace of the kernel. We propose an alternate method for obtaining properties that are needed for the kernel operator. Our main assumption is that the corresponding integration map $L_{\Gamma}$ is well-defined as an operator $L_{\Gamma}: Y(\mu ; \mathcal{K}) \rightarrow Y(\mu ; \mathcal{K})$, where $Y(\mu)$ is a 2-concave Banach function space having the Fatou property and such 
that $Y(\mu)^{\prime}$ is order continuous. This includes of course the case of Hilbert spaces $Y(\mu)=L^{2}(\mu)$-, but also for example the case of $Y(\mu)=L^{p}(\mu)$ for $1<p \leq 2$. Lorentz spaces $L^{p, q}(\mu)$ for $1<p \leq 2$ and certain Orlicz spaces are other examples.

Lemma 5.1. Let $Y(\mu)$ be a 2-concave Banach function space with the Fatou property such that $Y(\mu)^{\prime}$ is order continuous. Suppose that $T: Y\left(\mu ; \mathbb{C}^{n}\right) \rightarrow Y\left(\mu ; \mathbb{C}^{n}\right)$ is a bounded operator. Then the following assertions are equivalent.

(i) There is a constant $K>0$ such that

$$
\left\|\left(\sum_{i=1}^{n}\left\|T\left(f_{i}\right)\right\|^{2}\right)^{1 / 2}\right\| \leq K \sup _{\phi \in B_{Y\left(\mu ; \mathbb{C}^{n}\right)^{*}}}\left(\sum_{i=1}^{n}\left|\left\langle f_{i}, \phi\right\rangle\right|^{2}\right)^{1 / 2}
$$

for all $f_{1}, \ldots, f_{n} \in Y\left(\mu ; \mathbb{C}^{n}\right)$.

(ii) There are a function $0<g \in B_{\left(\left(Y(\mu)^{\prime}\right)_{[2]}\right)^{\prime}}$ and a probability measure $\eta \in\left(C\left(B_{Y\left(\mu ; \mathbb{C}^{n}\right)^{*}}\right)\right)^{\prime}$ such that for every $f \in Y\left(\mu ; \mathbb{C}^{n}\right)$,

$$
\int_{X} \frac{\|T(f)\|^{2}}{g} d \mu \leq K^{2} \int_{B_{Y\left(\mu ; \mathbb{C}^{n}\right)^{\prime}}}|\langle f, h\rangle|^{2} d \eta
$$

(iii) There is a factorization for $T$ as

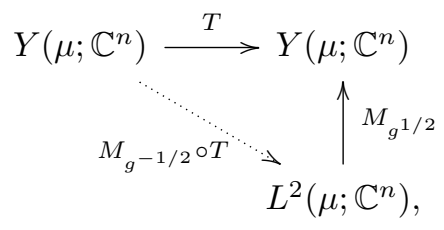

where $M_{g^{-1 / 2}} \circ T$ is 2-summing.

The proof is given again by an application of the Maurey-Rosenthal theory to the operator; essentially, it is a consequence of Theorem 2.5 in [23], but taking into account that the result provided there for scalar functions can be easily extended to the case of Köthe-Bochner spaces of vector valued functions. The use of homogeneous representation that is used in [8] gives the key for doing that (see also [10]). A relevant aspect of the proof is that it provides a function $g \in\left(\left(Y(\mu)^{\prime}\right)_{[2]}\right)^{\prime}$. Direct computations allow to relate this space with the space of multiplication operators $M\left(L^{2}(\mu), Y(\mu)\right)$. Indeed, note first that $Y(\mu)$ is 2-concave (we are always assuming that the corresponding with 2-concavity 
constant 1), and so $Y(\mu)^{\prime}$ is 2-convex (with 2-convexity constant equal to 1 ). Using the known equality $\left(Z(\mu)_{[p]}\right)^{\prime}=M\left(Z(\mu), L^{p}(\mu)\right)_{[p]}$ that holds for all $1 \leq p$ and can be found for example in [22, Ch.2] (see also [14] for a complete study of such kind of equalitites), we get

$$
\left(\left(Y(\mu)^{\prime}\right)_{[2]}\right)^{\prime}=\left(M\left(Y(\mu)^{\prime}, L^{2}(\mu)\right)\right)_{[2]}=M\left(L^{2}(\mu), Y(\mu)\right)_{[2]},
$$

where the Fatou property of $Y(\mu)$ and the order continuity of $Y(\mu)^{\prime}$ are used to obtain that $Y(\mu)^{\prime \prime}=Y(\mu)$ and the second equality above holds. Thus, $g^{1 / 2} \in M\left(L^{2}(\mu), Y(\mu)\right)$.

Proposition 5.2. Let $Y(\mu)$ be a 2-concave Banach function space with the Fatou property such that $Y(\mu)^{\prime}$ is order continuous. If for an operator $T: Y\left(\mu ; \mathbb{C}^{n}\right) \rightarrow Y\left(\mu ; \mathbb{C}^{n}\right)$ we have that (4) holds for all $f_{1}, \ldots, f_{n} \in Y\left(\mu ; \mathbb{C}^{n}\right)$ and a certain constant $K>0$, then there is a function $0<g \in B_{\left(\left(Y(\mu)^{\prime}\right)_{[2]}\right)^{\prime}}$ such that the operator $M_{g^{-1 / 2}} \circ T \circ M_{g^{1 / 2}}: L^{2}\left(\mu ; \mathbb{C}^{n}\right) \rightarrow$ $L^{2}\left(\mu ; \mathbb{C}^{n}\right)$ is well-defined and 2-summing. Consequently, it is a Hilbert-Schmidt (compact) operator.

Proof. As a consequence of Lemma 5.1, we get that there is a function $g$ that allows a factorization of $T$ in such a way that $M_{g^{-1 / 2}} \circ T: Y(\mu ; \mathcal{K}) \rightarrow L^{2}\left(\mu ; \mathbb{C}^{n}\right)$ is 2-summing. Since $g \in\left(M\left(L^{2}(\mu), Y(\mu)\right)\right)_{[2]}$, we have that $M_{g^{-1 / 2}} \circ T \circ M_{g^{1 / 2}}$ is well-defined from $L^{2}\left(\mu ; \mathbb{C}^{n}\right)$ to $L^{2}\left(\mu ; \mathbb{C}^{n}\right)$. The operator ideal property of 2 -summing operators ensures that the composition is 2-summing too, and so compact since Hilbert spaces are of course reflexive. Since Hilbert-Schmidt operators coincide with 2-summing operators on Hilbert spaces (see for example [9, Prop. 11.6]), we obtain the desired result.

We now show an example of space and an operator satisfying the conditions of the previous proposition.

Example 5.3. Consider the Lebesgue measure space $((-\pi, \pi], \mathcal{B}, \mu)$ with normalized measure $\mu$, and consider an orthonormal system $\left\{e_{k}\right\}$ of $L^{2}((-\pi, \pi])$ of functions that is uniformly bounded in $L^{\infty}((-\pi, \pi]) —$ for example, the trigonometric system- - Fixe also two complex sequences $\left(a_{k}\right),\left(b_{k}\right) \subseteq \ell^{1}(\mathbb{Z})$ and define the operator $S: L^{3 / 2}\left((-\pi, \pi] ; \mathbb{C}^{2}\right) \rightarrow$ 
$L^{2}\left((-\pi, \pi] ; \mathbb{C}^{2}\right)$ given by

$$
S(f):=\left(\sum_{k \in \mathbb{Z}} a_{k} e_{k}(\cdot)\left\langle f_{1}, e_{k}\right\rangle, \sum_{k \in \mathbb{Z}} b_{k} e_{k}(\cdot)\left\langle f_{2}, e_{k}\right\rangle\right), \quad f \in L^{3 / 2}\left((-\pi, \pi] ; \mathbb{C}^{2}\right),
$$

where, as usual, we write $f_{1}$ and $f_{2}$ for the $\mathbb{C}$-valued coordinate functions of $f$. Note that the operator is well defined as a consequence of a direct calculation using the pointwise boundedness of the elements of the system, the normalization of the measure $\mu$, Hölder's inequality and the absolute convergence of the series of $\left(a_{k}\right)$ and $\left(b_{k}\right)$.

Consider now a kernel operator $L_{\Gamma}: L^{2}\left((-\pi, \pi] ; \mathbb{C}^{2}\right) \rightarrow L^{2}\left((-\pi, \pi] ; \mathbb{C}^{2}\right)$ generated by a $\mathbb{M}_{2 \times 2}(\mathbb{C})$-reproducing kernel belonging for instance to the family described in Example 3.6 of [11]. By definition, $L_{\Gamma}$ is a Hilbert-Schmidt operator, and so 2-summing and compact. Note also that the inclusion map $\iota: L^{2}\left((-\pi, \pi] ; \mathbb{C}^{2}\right) \rightarrow L^{3 / 2}\left((-\pi, \pi] ; \mathbb{C}^{2}\right)$ is well-defined, and so continuous.

Our example is given by the map $T=\iota \circ L_{\Gamma} \circ S: L^{3 / 2}\left((-\pi, \pi] ; \mathbb{C}^{2}\right) \rightarrow L^{3 / 2}\left((-\pi, \pi] ; \mathbb{C}^{2}\right)$. The space $L^{3 / 2}((-\pi, \pi])$ is 2-concave, and the space and its dual are order continuous and have the Fatou property. By the ideal property of the 2 -summing operators, we get that $T$ is also 2 -summing. Moreover, for $f_{1}, \ldots, f_{n} \in L^{3 / 2}\left((-\pi, \pi] ; \mathbb{C}^{2}\right)$,

$$
\begin{aligned}
\left\|\left(\sum_{k=1}^{n}\left\|T\left(f_{k}\right)\right\|_{\mathbb{C}^{2}}^{2}\right)^{1 / 2}\right\|_{L^{3 / 2}((-\pi, \pi])} & \leq\|\iota\|\left\|\left(\sum_{k=1}^{n}\left\|L_{\Gamma} \circ S\left(f_{k}\right)\right\|_{\mathbb{C}^{2}}^{2}\right)^{1 / 2}\right\|_{L^{2}((-\pi, \pi])} \\
& =\|\iota\|\left(\sum_{k=1}^{n}\left\|L_{\Gamma} \circ S\left(f_{k}\right)\right\|_{L^{2}\left((-\pi, \pi] ; \mathbb{C}^{2}\right)}^{2}\right)^{1 / 2} \\
& \leq K\|\iota\| \sup _{\varphi \in B_{L^{3}\left((-\pi, \pi] ; \mathbb{C}^{2}\right)}}\left(\sum_{k=1}^{n}\left|\left\langle f_{k}, \varphi\right\rangle\right|^{2}\right)^{1 / 2}
\end{aligned}
$$

where $K$ stands for the 2-summability constant of the operator $L_{\Gamma} \circ S$. Therefore, inequality (4) is fullfilled. By Proposition 5.2 we can define a new compact operator as $M_{g^{-1 / 2}} \circ T \circ M_{g^{1 / 2}}: L^{2}\left((-\pi, \pi] ; \mathbb{C}^{2}\right) \rightarrow L^{2}\left((-\pi, \pi] ; \mathbb{C}^{2}\right)$ for a function $0<g \in$ $B_{\left(\left(L^{3 / 2}((-\pi, \pi])^{\prime}\right)_{[2]}\right)^{\prime}}=B_{L^{3}((-\pi, \pi])}$.

We present now the main result of this section. 
Theorem 5.4. (Weighted Mercer Theorem) Let $\mu$ be a finite measure Borel measure on the topological space $X$ with support equal to $X$. Suppose that the $\mathbb{M}_{n \times n}(\mathbb{C})$ reproducing kernel $\Gamma: X \times X \rightarrow \mathbb{M}_{n \times n}(\mathbb{C})$ is measurable. Let $Y(\mu)$ be a 2-concave Banach function space with the Fatou property such that $Y(\mu)^{\prime}$ is order continuous, and assume that the corresponding kernel operator is well-defined as a map $L_{\Gamma}: Y\left(\mu ; \mathbb{C}^{n}\right) \rightarrow Y\left(\mu ; \mathbb{C}^{n}\right)$ satisfying (4) for a certain $K>0$ and for every finite set of functions. Then the following assertions hold.

(i) There exists a real function $0<g$ with $g^{1 / 2} \in M\left(L^{2}(\mu), Y(\mu)\right)$ and such that the kernel $\Gamma$ defines a compact kernel operator $L_{\Gamma}^{g}: L^{2}\left(\mu / g ; \mathbb{C}^{n}\right) \rightarrow L^{2}\left(\mu / g ; \mathbb{C}^{n}\right)$ by means of the formula:

$$
L_{\Gamma}^{g}(f)=\int_{X} \Gamma(\cdot, y) f(y) \frac{d \mu(y)}{g(y)}, \quad f \in L^{2}\left(\mu / g ; \mathbb{C}^{n}\right) .
$$

(ii) There exists a countable family $\left\{f_{i}\right\}$ of $\mathbb{C}^{n}$-valued measurable functions that is an orthonormal system in $L^{2}\left(\mu ; \mathbb{C}^{n}\right)$ and for every $j \in \mathbb{N}$,

$$
L_{\Gamma}\left(g^{-1 / 2} f_{j}\right)=\sigma_{j} g^{1 / 2} f_{j},
$$

where $\left\{g^{1 / 2} f_{j}\right\}$ is an orthonormal basis of $\operatorname{ker}\left(L_{\Gamma}^{g}\right)^{\perp}-$ in $L^{2}\left(\mu / g ; \mathbb{C}^{n}\right)$.

(iii) For every $f \in L^{2}\left(\mu ; \mathbb{C}^{n}\right)$ and $x \in X$ we have that

$$
L_{\Gamma}\left(g^{-1 / 2} f\right)(x)=\sum_{j=1}^{\infty} \sigma_{j} g(x)^{1 / 2} \overline{f_{j}(x)} \int_{X}\left\langle f_{j}(y), \overline{f(y)}\right\rangle_{\mathbb{C}^{n}} d \mu(y)
$$

where the convergence of the series holds in $L^{2}\left(\mu / g ; \mathbb{C}^{n}\right)$.

Moreover, if $\int_{X} \operatorname{Tr} \Gamma(x, x) d \mu(x)<\infty$ and $\mathcal{H}_{\Gamma}$ is separable, then we also have that the functions $g^{1 / 2} f_{j}$ can be assumed to be continuous, and for all $x, y \in X$ and $k, l=1, \ldots, n$, the matrix valued kernel $\Gamma$ is given by

$$
\Gamma(x, y)_{k, l}=\sum_{i=1}^{\infty} \sigma_{j} g(x)^{1 / 2} \overline{f_{j}^{k}(x)} g(y)^{1 / 2} f_{i}^{l}(y)
$$

where the convergence is uniform on compact subsets of $X \times X$. Finally, $\left\{\sqrt{\sigma_{i}} g^{1 / 2} f_{j}\right\}$ is an orthonormal system of the associated space $\mathcal{H}_{\Gamma}$. 
Proof. (i) By using Lemma 5.1 there are a function $0<g \in B_{\left(\left(Y(\mu)^{\prime}\right)_{[2]}\right)^{\prime}}$-recall that this means that $g^{1 / 2} \in M\left(L^{2}(\mu), Y(\mu)\right)$ - and a probability measure $\eta \in\left(C\left(B_{Y\left(\mu ; \mathbb{C}^{n}\right)^{*}}\right)\right)^{\prime}$ satisfying

$$
\int_{X} \frac{\left\|L_{\Gamma}(f)\right\|^{2}}{g} d \mu \leq K^{2} \int_{B_{Y\left(\mu ; \mathbb{C}^{n}\right)^{\prime}}}|\langle f, h\rangle|^{2} d \eta, \quad f \in Y\left(\mu ; \mathbb{C}^{n}\right),
$$

for a certain positive constant $K$. In particular, this means that we can define the operator $L_{\Gamma}: Y\left(\mu ; \mathbb{C}^{n}\right) \rightarrow L^{2}\left(\mu / g ; \mathbb{C}^{n}\right)$, which is also 2-summing.

Note that, without loss of generality, we can assume that $g>\varepsilon$ for a fixed $\varepsilon>0$-just taking $g+\varepsilon$ instead of $g$ in the inequality above and taking into account that $(g+\varepsilon)^{1 / 2} \in$ $M\left(L^{2}(\mu), Y(\mu)\right)$ if $g^{1 / 2} \in M\left(L^{2}(\mu), Y(\mu)\right)$ - With this assumption $g^{-1} \in L^{\infty}(\mu)$-in particular the measure $d \mu / g$ is finite - and then we can define a multiplication operator $\widetilde{M}_{g^{-1}}: L^{2}(\mu / g) \rightarrow L^{2}(\mu / g)$ by the formula $\widetilde{M}_{g^{-1}}(f)=f g^{-1}$.

On the other hand, since the space $\left(\left(Y(\mu)^{\prime}\right)_{[2]}\right)^{\prime}$ can be identified with the 2-th power of the space of multiplication operators $M\left(L^{2}(\mu), Y(\mu)\right)$, then we have that $M_{g^{1 / 2}}: L^{2}(\mu) \rightarrow$ $Y(\mu)$ is well-defined and continuous. Note also that the operator $\widehat{M}_{g^{-1 / 2}}: L^{2}(\mu / g) \rightarrow$ $L^{2}(\mu)$ defines an isometry. Observe that these multiplication operators can be naturally defined for the corresponding spaces of $\mathbb{C}^{n}$-valued functions $L^{2}\left(\mu / g ; \mathbb{C}^{n}\right), L^{2}\left(\mu ; \mathbb{C}^{n}\right)$ and $Y\left(\mu ; \mathbb{C}^{n}\right)$.

Therefore, we can define the operator $S: L^{2}\left(\mu / g ; \mathbb{C}^{n}\right) \rightarrow L^{2}\left(\mu / g ; \mathbb{C}^{n}\right)$, given by $S(f)=$ $L_{\Gamma}(f)$ for functions $f \in L^{2}\left(\mu / g ; \mathbb{C}^{n}\right)$. Observe that $S$ can be written as $L_{\Gamma}^{g}=L_{\Gamma} \circ M_{g^{1 / 2}} \circ$ $\widehat{M}_{g^{-1 / 2}}$, that is, the following composition scheme holds for $S$ :

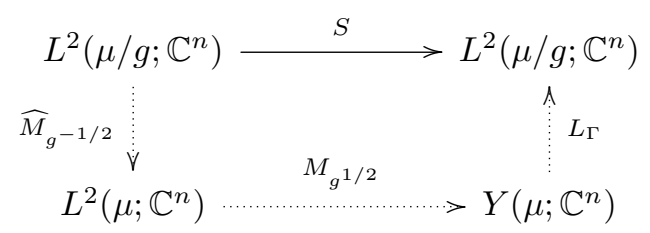

Consider now the operator $L_{\Gamma}^{g}=S \circ \widetilde{M}_{g_{-1}}: L^{2}\left(\mu / g ; \mathbb{C}^{n}\right) \rightarrow L^{2}\left(\mu / g ; \mathbb{C}^{n}\right)$. Note that this operator is the kernel operator associated to the (symmetric) $\Gamma$ when consider the integration with respect to the measure $\mu / g$. Hence the operator is selfadjoint and, of course, compact - since $S$ is-. 
(ii) Applying the Spectral Theorem to the compact selfadjoint operator $L_{\Gamma}^{g}$ we find an orthonormal system of eivenvectors $\left\{h_{j}\right\}$ of $L^{2}\left(\mu / g ; \mathbb{C}^{n}\right)$ associated to strictly positive eigenvalues $\left\{\sigma_{j}\right\}$. Define now the functions $f_{j}=g^{-1 / 2} h_{j}$. It is easy to see that they define an orthonormal system of $L^{2}\left(\mu ; \mathbb{C}^{n}\right)$, and for every $j \in \mathbb{N}$, and $x \in X$

$$
\begin{aligned}
L_{\Gamma}\left(g^{-1 / 2} f_{j}\right)(x) & =\int_{X} \Gamma(x, y) g(y)^{-1 / 2} f_{j}(y) d \mu(y) \\
& =\int_{X} \Gamma(x, y) h_{j}(y) \frac{d \mu(y)}{g(y)} \\
& =L_{\Gamma}^{g}\left(h_{j}\right)(x)=\sigma_{j} g(x)^{1 / 2} f_{j}(x) .
\end{aligned}
$$

Since $\left\{g^{1 / 2} f_{j}\right\}=\left\{h_{j}\right\}$, it is an orthonormal basis of $\operatorname{ker}\left(L_{\Gamma}^{g}\right)^{\perp}$-in $L^{2}\left(\mu / g ; \mathbb{C}^{n}\right)$.

(iii) Let us consider the matrix-valued kernels $\left\{\Gamma_{m}\right\}$ given by $\Gamma_{m}(x, y)=\left(\Gamma_{m}(x, y)_{k, l}\right)$ and

$$
\Gamma_{m}(x, y)_{k, l}:=\sum_{j=1}^{m} \sigma_{j} \overline{h_{j}^{k}(x)} h_{j}^{l}(y)=\sum_{j=1}^{m} \sigma_{j} g(x)^{1 / 2} \overline{f_{j}^{k}(x)} g(y)^{1 / 2} f_{j}^{l}(y),
$$

where $h_{j}^{k}$ and $h_{j}^{l}$ denote the corresponding $k$ th and $l$ th coordinate functions of the $\mathbb{C}^{n}$ valued function $h_{j}$. Note that the operator associated to $\Gamma_{m}$ is $L_{\Gamma_{m}}^{g}: L^{2}\left(\mu / g ; \mathbb{C}^{n}\right) \rightarrow$ $L^{2}\left(\mu / g ; \mathbb{C}^{n}\right)$, which is given by

$$
L_{\Gamma_{m}}^{g}(h)(x)=\sum_{j=1}^{m} \sigma_{j} g(x)^{1 / 2} \overline{f_{j}^{k}(x)} \int_{X} g(y)^{-1 / 2}\left\langle f_{j}(y), \overline{h(y)}\right\rangle_{\mathbb{C}^{n}} d \mu(y),
$$

for each $h \in L^{2}\left(\mu / g ; \mathbb{C}^{n}\right)$ and $x \in X$. Clearly, the limit of the associated kernel operators $L_{\Gamma_{m}}^{g}$ computed for each function $h$ defines a map that coincides with $L_{\Gamma}^{g}(h)$. In other words,

$$
\lim _{m} L_{\Gamma_{m}}^{g}(h)=L_{\Gamma}^{g}(h), \quad h \in L^{2}\left(\mu / g ; \mathbb{C}^{n}\right),
$$

where the convergence is in $L^{2}\left(\mu / g ; \mathbb{C}^{n}\right)$. Therefore, for each $f \in L^{2}\left(\mu ; \mathbb{C}^{n}\right)$

$$
\begin{aligned}
L_{\Gamma}\left(g^{-1 / 2} f\right) & =L_{\Gamma}^{g}\left(g^{1 / 2} f\right)=\lim _{m} L_{\Gamma_{m}}^{g}\left(g^{1 / 2} f\right) \\
& =\lim _{m} \sum_{j=1}^{m} \sigma_{j} g(x)^{1 / 2} \overline{f_{j}^{k}(x)} \int_{X} g(y)^{-1 / 2}\left\langle f_{j}(y), \overline{g(y)^{1 / 2} f(y)}\right\rangle_{\mathbb{C}^{n}} d \mu(y) \\
& =\sum_{j=1}^{\infty} \sigma_{j} g(x)^{1 / 2} \overline{f_{j}^{k}(x)} \int_{X}\left\langle f_{j}(y), \overline{f(y)}\right\rangle_{\mathbb{C}^{n}} d \mu(y),
\end{aligned}
$$


being the convergence in $L^{2}\left(\mu / g ; \mathbb{C}^{n}\right)$.

The last paragraph is a straightforward application of the results in [11] — Theorem 3.4 and Theorem $4.1-$ for the $L^{2}\left(\mu / g ; \mathbb{C}^{n}\right)$-orthonormal basis $\left\{h_{j}\right\}$ of $\operatorname{ker}\left(L_{\Gamma}^{g}\right)^{\perp}$ of eigenvectors of $L_{\Gamma}^{g}$, just observing that

$$
\int_{X} \operatorname{Tr} \Gamma(x, x) \frac{d \mu(x)}{g(x)}=\leq\left\|g^{-1}\right\|_{L^{\infty}(\mu)} \int_{X} \operatorname{Tr} \Gamma(x, x) d \mu(x)<\infty,
$$

since, as we have shown, we can assume that $g^{-1} \in L^{\infty}(\mu)$.

We can provide an easy example of application of this result with the help of the construction explained in Example 5.3.

Example 5.5. Let us consider the kernel operator $L_{\Gamma}: L^{2}\left((-\pi, \pi] ; \mathbb{C}^{2}\right) \rightarrow L^{2}\left((-\pi, \pi] ; \mathbb{C}^{2}\right)$, given in Example 3.6 of [11] by means of the $\mathbb{M}_{2 \times 2}(\mathbb{C})$-reproducing kernel:

$$
\Gamma(x, t)=\left(\begin{array}{cc}
\frac{(\pi-|t-x|)^{2}}{2} & \frac{\pi}{8}(t-x)(\pi-|t-x|) \\
\frac{\pi}{8}(x-t)(\pi-|x-t|) & \frac{(\pi-|t-x|)^{2}}{2}
\end{array}\right), \quad-\pi<t-x \leq \pi .
$$

The operator $L_{\Gamma}$ is Hilbert-Schmidt, and so 2-summing and compact. The eigvenvectors can be exactly computed in this case, as explained in Example 3.6 of [11]: they are given by the functions $f_{l, m}:(\pi, \pi] \rightarrow \mathbb{C}^{2}$ defined by $f_{l, m}(x)=\nu_{l, m} e^{i m x}$, for some constant vectors $\nu_{l, m} \in \mathbb{C}^{2}, m \in \mathbb{Z}, l=1,2$. The corresponding eigenvalues are $\sigma_{l, m}$ and, if we choose the eigenvalues that are strictly greater than 0 we get a basis of $\operatorname{ker}\left(L_{\Gamma}\right)^{\perp}$. This provides a decomposition of the kernel as

$$
\Gamma(x, t)=\sum_{l=1,2} \sum_{m \in \mathbb{Z}} \sigma_{l, m} \underbrace{\left(\begin{array}{ll}
\nu_{l, m}^{1} \overline{\nu_{l, m}^{1}} & \nu_{l, m}^{2} \overline{\nu_{l, m}^{1}} \\
\nu_{l, m}^{1} \overline{\nu_{l, m}^{2}} & \nu_{l, m}^{2} \overline{\nu_{l, m}^{2}}
\end{array}\right)}_{M_{l, m}} e^{i m(t-x)} .
$$

The reader can find a complete explanation of this example in [11].

Let us consider now the operators defined in Example 5.3 for the particular case of the sequences $a_{k}=b_{k}=1 / 2^{k}$ and the orthonormal system $e_{k}:(-\pi, \pi] \rightarrow \mathbb{C}$ defined by the trigonometric functions $e_{k}(x)=e^{-i k x}, x \in(-\pi, \pi]$. In this situation we can define 
an operator $T=\iota \circ L_{\Gamma} \circ S$ where

$$
S(f)=\left(\sum_{k \in \mathbb{Z}} \frac{1}{2^{k}} e^{-i k \cdot}\left\langle f_{1}, e^{-i k \cdot}\right\rangle, \sum_{k \in \mathbb{Z}} \frac{1}{2^{k}} e^{-i k \cdot}\left\langle f_{2}, e^{-i k \cdot}\right\rangle\right), \quad f \in L^{3 / 2}\left((-\pi, \pi] ; \mathbb{C}^{2}\right) .
$$

Using (5) and the orthonormality of the trigonometric system, we obtain

$$
\begin{aligned}
T(f)(x) & =L_{\Gamma}\left(\sum_{k \in \mathbb{Z}} \frac{1}{2^{k}} e^{-i k \cdot}\left\langle f_{1}, e^{-i k \cdot}\right\rangle, \sum_{k \in \mathbb{Z}} \frac{1}{2^{k}} e^{-i k \cdot}\left\langle f_{2}, e^{-i k \cdot}\right\rangle\right)(x) \\
& =\int_{(-\pi, \pi]}\left(\sum_{l=1,2} \sum_{m \in \mathbb{Z}} \sigma_{l, m} M_{l, m} e^{i m(t-x)}\right)\left(\sum_{k \in \mathbb{Z}} \frac{1}{2^{k}} e^{-i k t}\left(\begin{array}{c}
\left\langle f_{1}, e^{-i k \cdot}\right\rangle \\
\left\langle f_{2}, e^{-i k \cdot}\right\rangle
\end{array}\right)\right) d \mu(t) \\
& =\left(\sum_{l=1,2} \sum_{m \in \mathbb{Z}} \frac{\sigma_{l, m}}{2^{m}} M_{l, m} e^{-i m x}\left(\begin{array}{c}
\left\langle f_{1}, e^{-i m \cdot}\right\rangle \\
\left\langle f_{2}, e^{-i m \cdot}\right\rangle
\end{array}\right)\right) \\
& =\int_{(-\pi, \pi]}\left(\sum_{l=1,2} \sum_{m \in \mathbb{Z}} \frac{\sigma_{l, m}}{2^{m}} M_{l, m} e^{i m(t-x)}\right) f(t) d \mu(t),
\end{aligned}
$$

for all $f \in L^{3 / 2}\left((-\pi, \pi] ; \mathbb{C}^{2}\right)$ and $x \in(-\pi, \pi]$. Therefore, $T$ is actually a kernel operator associated to

$$
\widetilde{\Gamma}(x, t)=\sum_{l=1,2} \sum_{m \in \mathbb{Z}} \frac{\sigma_{l, m}}{2^{m}} M_{l, m} e^{i m(t-x)},
$$

which is clearly a $\mathbb{M}_{2 \times 2}(\mathbb{C})$-reproducing kernel.

Acknowledgments. The authors thank the referees for their useful suggestions, which helped to improve the content of the paper.

\section{REFERENCES}

[1] L. Agud, J. M. Calabuig and E. A. Sánchez Pérez, The weak topology on qconvex Banach function spaces, Mathematische Nachrichten 285 (2012) 136-149.

[2] C. Carmeli, E. De Vito, and A. Toigo, Vector valued reproducing kernel Hilbert spaces of integrable functions and Mercer theorem, Analysis and Applications 4(04) (2006) 377-408.

[3] C. Carmeli, E. De Vito, A. Toigo, and V. Umanità, Vector valued reproducing kernel Hilbert spaces and universality, Analysis and Applications 8(01) (2010) 19-61.

[4] P. Cembranos and J. Mendoza, Banach spaces of vector-valued functions, Springer, Berlin 2006.

[5] A. Christmann, F. Dumpert and D.-H. Xiang., On extension theorems and their connection to universal consistency in machine learning, Analysis and Applications 14 (2016) 795-808. 
[6] J. Cerdà, H. Hudzik and M. Mastylo, Geometric properties of Köthe-Bochner spaces. Math. Proc. Cambridge Philos. Soc. 120 (3) (1996) 521-533.

[7] Chavan, S., Podder, S. and and Trivedi, S. . Commutants and reflexivity of multiplication tuples on vector-valued reproducing kernel Hilbert spaces. J. Math. Anal. Appl. 466 (2018) 1337-1358.

[8] A. Defant, Variants of the Maurey-Rosenthal theorem for quasi Köthe function spaces, Positivity, 5 (2001) 153-175.

[9] A. Defant and K. Floret, Tensor norms and operator ideals, Elsevier, Amsterdam 1992.

[10] A. Defant and E. A. Sánchez Pérez, Maurey-Rosenthal factorization of positive operators and convexity, J. Math. Anal. Appl. 297 (2004) 771-790.

[11] E. De Vito, V. Umanità, and S. Villa, An extension of Mercer theorem to matrix-valued measurable kernels, Applied and Computational Harmonic Analysis 34 (3) (2013) 339-351.

[12] M. Eigel, and K. Sturm, Reproducing kernel Hilbert spaces and variable metric algorithms in PDEconstrained shape optimization, Optimization Methods and Software (2017) 1-29.

[13] G.E. Fasshauer, F. J. Hickernell and Q. Ye. Solving support vector machines in reproducing kernel Banach spaces with positive definite functions. Applied and Computational Harmonic Analysis 38 (2015) 115-139.

[14] O. Galdames Bravo, Generalized Köthe $p$-dual spaces, Bull. Belgian Math. Soc.-Simon Stevin 21.2 (2014) 275-289.

[15] E. Hille and R.S. Phillips, Functional analysis and semigroups, Amer. Math. Soc., Providence 1957.

[16] H. Kadri, E. Duflos, P. Preux, S. Canu, A. Rakotomamonjy, and J. Audiffren, Operator-valued kernels for learning from functional response data. The Journal of Machine Learning Research, 17(1) (2016) 613-666.

[17] J. L. Krivine, Thèorèmes de factorisation dans les espaces rèticulès, Sèminaire Maurey-Schwartz 1973-1974: Espaces L $L^{p}$, applications radonifiantes et gèomètrie des espaces de Banach, Exp. Nos. 22 et 23. Centre de Math., ècole Polytech., Paris, (1974).

[18] P.K. Lin, Köthe-Bochner function spaces, Birkhauser, Boston 2004.

[19] J. Lindenstrauss and L. Tzafriri, Classical Banach Spaces II, Springer, Berlin 1979.

[20] P. Meyer-Nieberg, Banach lattices, Springer, Berlin 1991.

[21] H.Q. Minh, L. Bazzani and V. Murino. A unifying framework in vector-valued reproducing kernel Hilbert spaces for manifold regularization and co-regularized multi-view learning. The Journal of Machine Learning Research 17 (2016) 769-840.

[22] S. Okada, W. J. Ricker and E. A. Sánchez Pérez, Optimal Domain and Integral Extension of Operators acting in Function Spaces, Operator Theory: Adv. Appl., vol. 180, Birkhäuser, Basel 2008. 
[23] C. Palazuelos, E. A. Sánchez Pérez, and P. Tradacete, Maurey-Rosenthal factorization for psumming operators and Dodds-Fremlin domination, Journal of Operator Theory 68(1) (2012) 205222.

[24] I. Steinwart and A. Christmann, Support vector machines. Springer, Berlin 2008.

[25] Y. Xu and Y. Qi, Generalized Mercer kernels and reproducing kernel Banach spaces. Memoirs of the American Mathematical Society. Vol.258 n.1243, 2019.

[26] H. Zhang, Y. Xu, and Q. Zhang, Refinement of operator-valued reproducing kernels, Journal of Machine Learning Research 13 (2012) 91-136.

[27] H. Zhang and J. Zhang, Vector-valued reproducing kernel Banach spaces with applications to multitask learning. Journal of Complexity 29 (2013) 195-215.

L. Agud, Departamento de Matemática Aplicada, Universitat Politècnica de València, Campus de Alcoy, Valencia. Spain

E-mail address: lagudal@mat.upv.es

J.M. Calabuig, E. A. Sánchez Pérez, Instituto Universitario de Matemática Pura y ApliCada, Universitat Politècnica de València, Camino de Vera s/n, 46022 Valencia. Spain.

E-mail address: jmcalabu@mat.upv.es, easancpe@mat.upv.es 